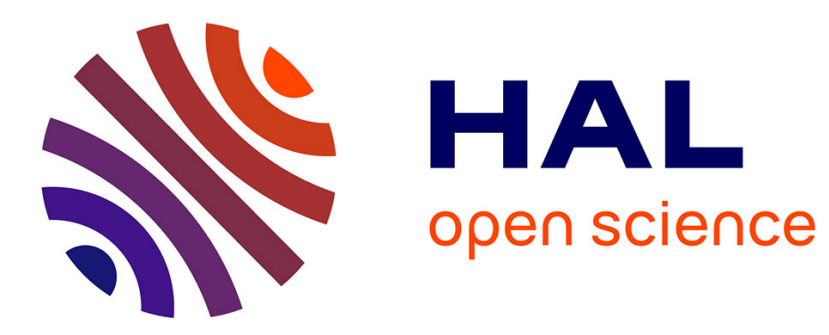

\title{
Mineral resources for renewable energy: Optimal timing of energy production
}

\author{
Adrien Fabre, Mouez Fodha, Francesco Ricci
}

\section{To cite this version:}

Adrien Fabre, Mouez Fodha, Francesco Ricci. Mineral resources for renewable energy: Optimal timing of energy production. Resource and Energy Economics, 2020, 59, 10.1016/j.reseneeco.2019.101131 . hal-02446805

\section{HAL Id: hal-02446805 \\ https://hal.science/hal-02446805}

Submitted on 21 Jan 2020

HAL is a multi-disciplinary open access archive for the deposit and dissemination of scientific research documents, whether they are published or not. The documents may come from teaching and research institutions in France or abroad, or from public or private research centers.
L'archive ouverte pluridisciplinaire HAL, est destinée au dépôt et à la diffusion de documents scientifiques de niveau recherche, publiés ou non, émanant des établissements d'enseignement et de recherche français ou étrangers, des laboratoires publics ou privés. 


\title{
Mineral Resources for Renewable Energy:
}

\section{Optimal Timing of Energy Production*}

\author{
Adrien Fabreł Mouez Fodha ${ }^{\ddagger}$ and Francesco Ricci ${ }^{\S}$
}

Resource and Energy Economics Vol. 59, 101131

https://doi.org/10.1016/j.reseneeco.2019.101131

Submitted: February 7, 2019. Accepted: November 4, 2019

\begin{abstract}
The production of energy from renewable sources is much more intensive in minerals than that from fossil resources. The scarcity of certain minerals limits the potential for substituting renewable energy for scarce fossil resources. However, minerals can be recycled,while fossil resources cannot. We develop an intertemporal model to study the dynamics of the optimal energy mix in the presence of mineral intensive renewable energy and fossil energy. We analyze energy production when both mineral and fossil resources are scarce,but minerals are recyclable. We show that the greater the recycling rate of minerals, the more the energy mix should rely on renewable energy, and the sooner should investment in renewable capacity take place. We confirm these results even in the presence of other better known factors that affect the optimal schedule of resource use: expected productivity growth in the renewable sector, imperfect substitution between the two sources of energy, convex extraction costs for mineral resources and pollution from the use of fossil resources.
\end{abstract}

Keywords : Renewable and Non-Renewable Natural Resources; Mineral Resources; Recycling; Energy Transition.

JEL CODES: Q3, Q2, Q42, Q54.

${ }^{*}$ We are grateful for helpful comments to participants at FAERE 2015, WCERE 2018, SURED 2018 conferences and the International Conference on Environmental Economics at University of Orléans in 2018, BETA, CEE-M and EconomiX seminars, in particular Olivier Vidal and Katheline Schubert. We are very grateful to the anonymous reviewers for valuable comments. Fodha and Ricci acknowledge financial support from CNRS Mission Interdisciplinarité federative research program ReMinER, Ricci benefited as well of support from ANR REVE (ANR 14-CE05-0008-02).

${ }^{\dagger}$ Paris School of Economics and University Paris 1 Panthéon Sorbonne. adrien.fabre@psemail.eu.

${ }^{\ddagger}$ Paris School of Economics and University Paris 1 Panthéon Sorbonne. mouez.fodha@univ-paris1.fr.

${ }^{\S}$ CEE-M, Univ de Montpellier, CNRS, INRA, Montpellier SupAgro. francesco.ricci@umontpellier.fr. 


\section{Introduction}

Renewable sources of energy are generally more scattered than non renewable ones. In particular this is the case of wind or solar energy, as compared to coal or gas. More infrastructure to capture these renewable sources, and therefore a larger quantity of mineral inputs is required to produce one unit of final energy from renewable than from non renewable sources of energy. ${ }^{1}$ For instance, Hertwich et al. (2015) conclude that one unit of electricity requires "11-40 times more copper for photovoltaic systems and 6-14 times more iron for wind power plants", than from conventional fossil generation, as one can see in Figure 1. Concern about mineral intensity of renewable sources of energy has been expressed in official reports and academic studies. ${ }^{2}$

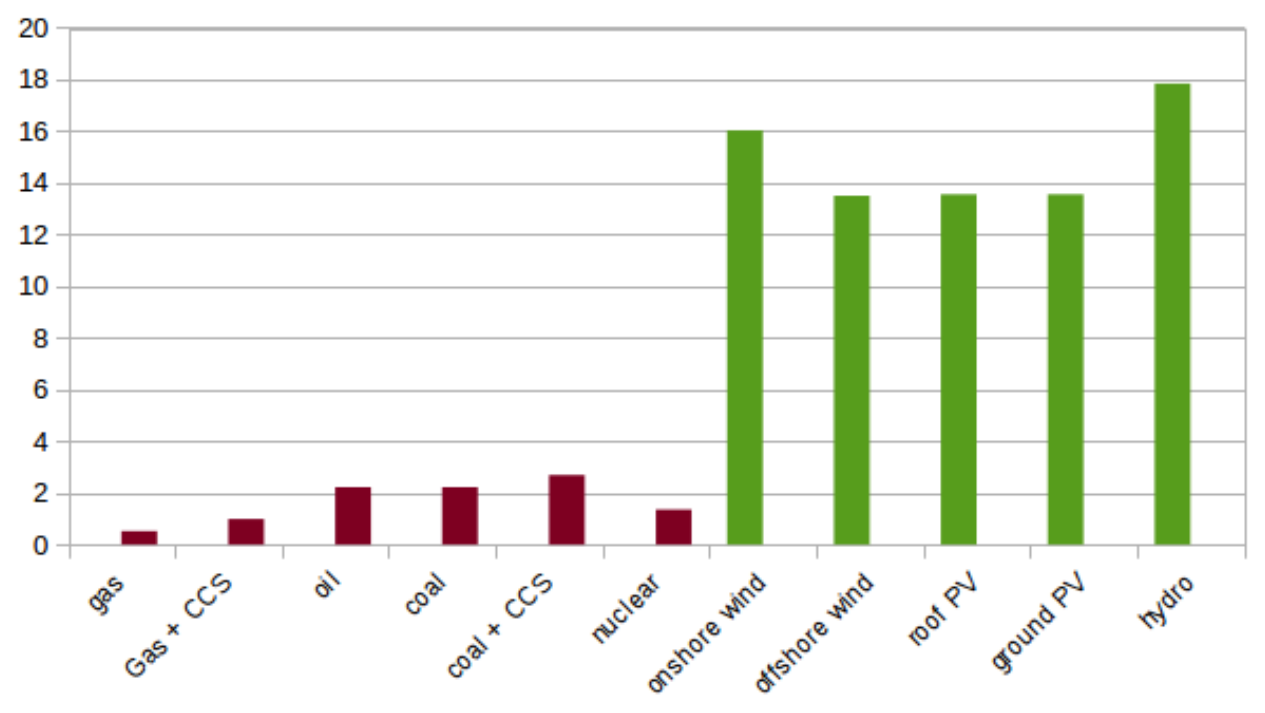

Figure 1: Copper intensity of energy technologies, kg/MWh, from Hertwich et al. (2015)

The objective of this paper is to study how the schedule of energy production depends on mineral resources, as scarce inputs in the production of renewable energy. We present a theoretical model and bring along a novel argument in favor of early development of the production capacity for energy from renewable sources, which relies on the asymmetry between the types of natural resources used to produce energy services. When a unit of non renewable resource is directly used as fuel to supply energy services through combustion, as in the case of oil, gas and coal, that amount of resource is definitely lost. When a unit of mineral resources is embedded in the equipment and infrastructure used to produce energy from renewable sources, it supplies a flow of energy services over an interval of time and, at the end of the life cycle of the equipment, it adds to the stock of secondary mineral resources that can be recycled. Hence some part of the original unit of resource can provide services in the next period: the higher the recycling rate, the less one needs to extract minerals in the future.

\footnotetext{
${ }^{1}$ In the case of intermittent renewable energy, backup or storage capacity requirements exacerbate this difference in mineral intensity.

${ }^{2}$ See for instance Vidal et al. (2013), Moss et al. (2013), Ali et al. (2017), Vidal et al. (2017), as well as Arrobas et al. (2017), European Commission (2017), European Commission (2018), DOE (2013), and the U.S. Presidential executive order on the Strategy to ensure secure and reliable supplies of critical minerals (Dec. 2017).
} 
If minerals were not recyclable, they could not be reused — just as fossil resources — and the two types of resources would be analogous.

While the opportunity to recycle a non renewable natural resource improves the production possibilities set of the economy, it also requires time as an input in order to do so. From a technological perspective, recycling first requires to use the primary (currently extracted) resource, in order to build, with some delay, the secondary (recycled) resource. This technological constraint interacts with social preferences in determining the optimal schedule of resource extraction and use. To illustrate this, let us consider a society, with no preference for the present, where neither extraction nor recycling are costly, that wishes to maintain the level of resource use constant at a given level over a finite interval of time. If it is endowed with a stock of a non recyclable resource, it should spread it evenly over the planning horizon. If instead the resource can be partially recycled, say at a recovery rate $\delta \in(0,1)$, with some time lag, say ten years, it should use exclusively primary resources during the first ten years, then reduce the extraction by the rate $1-\delta$ during the following decades. As compared to the former case, the intertemporal profile of resource extraction is brought forward.

Together, the technological specificity of recycling mineral resources and the relative mineral intensity of renewable energy provide a rationale for developing more renewable energy infrastructure in the initial period than in subsequent ones and to choose a larger share for renewables in the energy mix, as compared to a case without recycling. Our analysis is based on a simplified description of the economic problem.

In our model, agents value energy services which result from a combination of energy provided by two distinct sources: the flow of renewable energy and combustion of a non renewable fossil resource. These sources are more or less good substitutes, either because of heterogeneous uses (Chakravorty and Krulce, 1994) or because of the intermittent availability of the renewable sources (Ambec and Crampes, 2019). The production of renewable energy employs specific equipment, dubbed "green" capital, embedding mineral non renewable resources. Part of the mineral resources embedded in the current period equipment can be also used in the next periods. The reserves of the two non renewable resources (fossils and minerals) are scarce.The issue is the timing of their extraction that maximizes the net present value of the utility from energy services.

The answers we obtain encompass some well known arguments, as for instance that the development of renewables should be postponed in the expectations of a productivity improvement of green capital. But the framework we consider allows us to put forward two original arguments: to the extent that mineral resources embedded in that equipment and infrastructure can be recycled, the development of renewable energy should be brought forward in time, and the energy mix should rely largely on renewable sources.

The assumption of a finite and scarce supply of minerals to build up the stock of green capital allows us to pinpoint these novel arguments, which rely on the intertemporal dependence in the use of the two non renewable resources. The analysis would be affected if we were to consider competition in the use of the global supply of minerals between investment in green capital and other uses. For instance, in the extreme opposite case, one can assume that the demand for minerals from the energy sector is so small that it does not affect their equilibrium price. Minerals for investment in 
green capital would then be available at some exogenous marginal cost, breaking the intertemporal dependence of green capital investment decisions. ${ }^{3}$ Let us emphasize that this is the case in most of the literature, where there is no direct intertemporal linkage of renewable energy production through scarcity of embedded non-renewable resources. The plausible case lies in between this extreme and our framework. Thus the mechanism we point out shall be at work, though its importance should be evaluated empirically.

Other factors can affect the optimal decision on the timing of investment in green capital. In particular, mineral extraction should be delayed when endowment in green capital is excessive. ${ }^{4}$ In the two-period version of our model, we consider several applications and confirm that our original results hold despite the presence of alternative mechanisms. Choices related to the intertemporal allocation of scarce resources crucially depend on social preferences, specifically the willingness to smooth consumption over time. A first factor determining the timing of resource use is the expected pace of improvement in the productivity of green capital. Faster expected productivity growth tends to postpone investment in green capital, if the willingness to smooth consumption is high enough. Yet the asymmetry across resources in terms of recyclability still calls for early investment in green capital. A second factor is the degree of substitutability between the two sources of energy. Due to their physical properties or to intermittency, they are considered more or less good substitutes, or even complements, in providing energy services. We show that the higher the substitutability, the larger the marginal effects of recyclability on initial green investment and the share of renewables in the energy mix. In other words, the more flexible is the technology, the more society takes advantage of the opportunity opened by recycling. This is of special interest given that, under current technology, substitutability between conventional and renewable energy relies on storage and that electricity storage capacity is particularly intensive in minerals. A third factor we consider is the convex nature of resource extraction costs. This consideration points at the benefit of spreading resource use over time. Yet, even in the presence of convex extraction costs, an improvement in recyclability calls for earlier use of minerals, thus fostering green investment. Finally, a major rationale for early investment in green capital is based on the objective to substitute for the use of fossil energy sources, because it generates pollution. Also in this case improved recyclability fosters early green investment. Moreover it boosts the share of renewables in the energy mix over both periods and a reduction in total polluting emissions (i.e. total fossil resource use) for sufficiently low willingness to smooth consumption.

Our work is related to several strands of the literature. The analytical approach focuses on the efficient management à la Hotelling (1931) of two types of non renewable resources, fossil and minerals (Heal, 1993). Much attention has been paid to the case of perfect substitutes, to study

\footnotetext{
${ }^{3}$ Moreover, assuming a small role of the energy sector on the market for primary mineral resources, implies that it cannot affect the market for secondary mineral resources, ruling out of the analysis any potential impact of the efficiency of the recycling technology on the timing of energy production.

${ }^{4}$ This endowment results from investment before the start of optimal regulation. In principle it may exceed what the optimal regulator would have chosen. In this case, the regulator would choose to rely initially only on the endowment and then, for a few initial periods, only on its recycled part, before beginning to extract minerals to add to this part. We consider this case reminiscent of investment in photovoltaic capacity in Spain, although it does not seem empirically plausible (see discussion at the end of Section 3).
} 
the optimal order of extraction. ${ }^{5}$ Instead, we actually consider the case of simultaneous use of the two sources of energy, conventional and renewable, in the spirit of growth theory applied to the energy transition. ${ }^{6}$ Moreover, our results do not rely on the effect of scarcity on extraction costs and are derived in a deterministic framework. ${ }^{7}$ Our contribution consists of an original argument concerning the optimal timing of investment in green capital, used to produce energy from renewable sources. This is related to an extensive literature covering the policies associated with the energy transition. Among the wealth of arguments that have been put forward, some of which discussed in the previous paragraph, we recall the following. Amigues et al. (2015) point out that, in the presence of capital adjustment costs, investment to build the infrastructure for the production of renewable energy should begin early on and be spread out over time. Vogt-Schilb et al. (2018) argue that early investment in green capital is particularly valuable in the energy sector because of the long-lived nature of such capital. Lemoine and Traeger (2014) explain how uncertainty and irreversibility, due to lagged damages and investment, together affect the optimal timing of pollution abatement. ${ }^{8}$ Technological progress resulting from learning-by-doing calls for early investment (Kverndokk and Rosendahl, 2007). As put forward in Goulder and Schneider (1999), the optimal investment in carbon free capital is affected by the fact that $R \& D$ expenditure can be targeted to such technologies. Boosting early investment may be essential to trigger sufficient R\&D to escape a lock-in in the polluting technology (Acemoglu et al., 2012). This rich literature adequately examines different aspects of the timing of the energy transition, yet none of them embeds the dependency of renewable production on recyclable but scarce minerals.

In our analysis, recycling is crucial for the results. The efficient paths of resource extraction and recycling are considered as early as Weinstein and Zeckhauser (1973), Schulze (1974) or Dasgupta and Heal (1979). In economies confronted to the limited availability of resources, recycling reduces the reliance on primary resources and postpones the extraction of resources. This result is extended in various dimensions, by taking into account the material balance constraint (Pittel et al., 2010) or technological progress (Di Vita, 2001). A more recent literature considers that recycling, by linking past and current production, may generate economic cycles (De Beir et al., 2010; Fodha and Magris, 2015; Boucekkine and El Ouardighi, 2016). Finally, some articles focus on market failures associated with missing markets for waste and the resulting pollution (Hoel, 1978; Musu and Lines, 1995). However, none of these works considers the role of recycling in the interplay between exhaustible resources and energy production. As we show hereafter, recycling of minerals

\footnotetext{
${ }^{5}$ See in particular the "least cost first" principle in Herfindahl (1967) and its qualifications (Kemp and Van Long, 1980; Lewis, 1982; Amigues et al., 1998). The case of imperfect substitution across non renewable resources is considered in Wirl (1988) and Chakravorty and Krulce (1994). Also the case of renewable resources has longtime been studied as a permanent shift to a perfect substitute (Tahvonen and Salo, 2001; Tsur and Zemel, 2005).

${ }^{6}$ For instance Smulders and de Nooij (2003) or Grimaud and Rouge (2008) -where the labor supply is equivalent to a constant flow of renewable energy-, Pittel and Bretschger (2010), Hart (2019).

${ }^{7}$ This differs from much of the related literature: the stochastic framework is used to analyze R\&D investment to introduce an abundant substitute to the non renewable resource (Davison, 1978; Kamien and Schwartz, 1978; Dasgupta et al., 1982), and the stock effect on extraction cost in the analysis of the optimal switching to a backstop technology (Oren and Powell, 1985 and citations therein).

${ }^{8}$ Uncertainty and irreversibility is addressed in a microeconomic perspective in Murto and Nese (2002) and Wickart and Madlener (2007), where a firm optimally chooses the timing for investing in one of two alternative energy technologies.
} 
is relevant to the transition to a low carbon economy, given that "the world cannot tackle climate change without adequate supply of raw materials to manufacture clean technologies" (Ali et al., 2017).

We present our model in section 2. Section 3 presents the analysis and the results of the benchmark case, with infinite horizon and specific functional forms for the utility and the production functions. Then, we consider in section 4 further issues in a two-period version of the model. First, we check that the main results hold in this version, then we consider differences in the productivity growth across the two energy types. Second, we study the role of the degree of substitutability between energy sources in the production of energy services. Third, we introduce convex extraction costs for mineral resources. Finally, we take into account environmental damages from the use of fossil resources. To conclude we give some perspectives, in particular on the determinants of the recycling rate, from which we abstract in this paper.

\section{The model}

We study an economy in discrete time, where periods are denoted by $t \in \mathbb{N}_{0} \cdot{ }^{9}$ Let us consider a representative household, whose utility is a function of consumption of energy services $q_{t}:^{10}$

$$
u\left(q_{t}\right)
$$

with $u^{\prime}>0, u^{\prime \prime} \leq 0$.

Energy services combine two flows: energy from non renewable resources, $x_{t}$, and energy from renewable sources, $y_{t}$. Formally we write:

$$
q_{t}=Q\left(x_{t}, y_{t}\right)
$$

with $Q_{i}^{\prime}>0, Q_{i}^{\prime \prime} \leq 0 i \in\{x, y\}$. The degree to which the two types of energy can be combined to produce energy services may vary from perfect substitutability to perfect complementarity. ${ }^{11}$

The energy flow $x_{t}$ is produced transforming the quantity of extracted non renewable resource $f_{t} \geq 0$, which we dub fossil resources, according to the linear production function:

$$
x_{t}=A_{t} f_{t}
$$

\footnotetext{
${ }^{9}$ With a slight abuse of notation, for two dates $t_{2}>t_{1} \geq 0$ we write $t \in\left[t_{1}, t_{2}\right]$ to refer to $t \in\left[t_{1}, t_{2}\right] \cap \mathbb{N}_{0}$ or $t \in\left\{t_{1} ; t_{1}+1 ; \ldots ; t_{2}\right\}$. Similarly, we simply write $t \geq 0$ for $t \in \mathbb{N}_{0}$.

${ }^{10}$ For the moment we abstract from any influence on the household's utility from the energy system. In section 4 we assume that utility also depends on the types of energy sources that are used to produce energy services, namely that the use of one source also generates disutility due to pollution.

${ }^{11}$ Two approaches can be considered. Either firms sell energy services by using the two types of energy. This is the case, for instance, of a power company generating electricity out of a differentiated portfolio of power stations, some based on conventional fossil resources, others on wind and solar power. Alternatively, one can consider that households directly consume the two resources. For instance, a household endowed of a solar thermal panel and a gas fueled heater to heat water, can use the two sources of energy as imperfect substitutes due to the intermittent nature of the former. We analyze the role of this assumption in section 4.2.
} 
where $A_{t}$ is the exogenous productivity index. Resource extraction is costless. ${ }^{12}$ The quantity of fossil resources is limited, it is initially available in a finite stock $F$ and is directly reduced by extraction:

$$
F \geq \sum_{t \geq 0} f_{t}
$$

The flow of renewable energy $y_{t}$ is produced employing a specific stock of capital $K_{t}$, which we dub "green" capital, according to the linear technology:

$$
y_{t}=B_{t} K_{t}
$$

where $B_{t}$ is the exogenous productivity index. Green capital is built out of minerals. Specifically, the capital stock at date $t$ is the sum of minerals extracted at date $t$ - the primary resource $m_{t}$ - and the stock of secondary minerals recycled from previous period's green capital $\delta K_{t-1}$. The exogenous parameter $\delta \in[0,1]$ measures the rate at which minerals embedded in the capital stock can be recycled from one period to the next. We implicitly assume perfect substitutability between primary and recycled mineral resources, and the possibility of infinite recycling. ${ }^{13}$ Defining $K_{-1} \geq 0$ as the stock of minerals embedded in the capital stock before date 0 , and assuming a constant recycling rate, the history of mineral extraction determines the stock of green capital: ${ }^{14}$

$$
K_{t}=K_{-1} \delta^{t+1}+\sum_{\tau=0}^{t} m_{\tau} \delta^{t-\tau} .
$$

Notice that with exogenous efficiency of the recycling technology, the parameter $\delta$ can be interpreted as the complement of the depreciation factor of capital in a standard accumulation process. An increase in the recycling rate could be equivalent to a decrease in the depreciation rate. Nevertheless, in our case, investment here consists of mineral resources, differently from the standard notion of capital. ${ }^{15}$ Therefore, green capital is limited by a threshold determined by the total stock of resources. Minerals are non-renewable resources, initially available in a finite stock $M$. Primary

\footnotetext{
${ }^{12}$ We consider costly extraction in section 4.3 .

${ }^{13}$ In this article, we do not take into account the cost of waste recovery and processing and the lower quality of recycled resources. Di Vita (2007) takes into account imperfect substitutability between the non-renewable resource and recycled waste in the production process. He analyzes the economic growth rate and the time profile of resource extraction. Lafforgue and Rouge (2019) assume that the quality of recycled materials evolves and could make them ultimately unproductive. Like these authors and much of the literature, we also restrict our analysis to the case of an exogenous recycling rate.

${ }^{14}$ In practice, both types of energy sources require specific capital embedding some mineral resources. Our focus is the asymmetry in mineral intensity between the specific capital for each energy source. We therefore adopt the extreme assumption that only one energy source relies on the specific capital, so as to simplify the analysis, without loosing in the qualitative features of our model.

${ }^{15}$ In the standard approach, investment results from non consumed output. In our setting this could consist of energy services not devoted to their consumption $q_{t}$. Instead under our assumption, the stock of green capital consists of a stock of productive mineral resources.
} 
extraction is constrained over time by ${ }^{16}$

$$
M \geq \sum_{t \geq 0} m_{t}
$$

In our framework the distinction between fossil and renewable sources of energy hinges on the recycling rate of minerals $\delta$. If minerals were perfectly recyclable, i.e. $\delta=1$, it would be possible to produce forever a flow $B_{t} M$ of renewable energy, once the specific equipment had been installed at its maximum potential. If minerals were not recyclable, i.e. $\delta=0$, they could not be used twice - just as fossil resources - and the two types of resources would be analogous.

We analyze optimal trajectories, assuming that a benevolent planner chooses the path of resource extraction that maximizes intertemporal discounted utility of the representative household, subject to technology constraints and resource dynamics. It applies a social pure discount rate $\rho>0$ and solves the following problem

$$
\begin{aligned}
(\mathcal{P}): & \max _{f_{t}, m_{t}} \sum_{t \geq 0} \frac{1}{(1+\rho)^{t}} u\left(q_{t}\right) \\
& \text { subject to }(2.2)-(2.7) \text { and } f_{t}, m_{t} \geq 0 \\
& \text { with } M, F, K_{-1} \text { given. }
\end{aligned}
$$

\section{Optimal energy production with infinite horizon}

In this section, we further specify the production and utility functions, in order to be able to characterize the optimal policy by closed-form solutions. Specifically, we assume a unitary elasticity of substitution between fossil and renewable energy

$$
Q\left(x_{t}, y_{t}\right)=x_{t}^{\alpha} y_{t}^{1-\alpha}
$$

with $\alpha \in(0,1)$. Moreover we restrict the analysis to the case of constant and equal productivity, and set $\forall t A_{t}=B_{t}=1$. We also assume a utility function with a constant elasticity of intertemporal substitution of consumption ${ }^{17}$

$$
u\left(q_{t}\right)=\frac{1}{1-\varepsilon} q_{t}^{1-\varepsilon}
$$

with $\varepsilon>0$.

These assumptions imply that the extraction of fossil resources is always positive, i.e. $\forall t, f_{t}>0$. In fact, if $f_{t}=0$ at some $t, q_{t}=0$, which is suboptimal since the marginal utility of $q$ is infinite at $q=0$. The reasoning applies to green capital, so that $\forall t, K_{t}>0$. The same argument applies

\footnotetext{
${ }^{16}$ As explained in the Introduction, this is a crucial assumption for our analysis. The results concerning the role of the mineral recycling rate for initial green investment and the energy mix change drastically if, instead of (2.7), one considers a perfectly elastic supply of $m_{t}$ at some exogenous marginal cost representing the relative intensity in minerals of renewable energy production as compared to conventional energy.

${ }^{17}$ The elasticity of intertemporal substitution of consumption equals $1 / \varepsilon$. For $\varepsilon=1, u\left(q_{t}\right)=\ln q_{t}$.
} 
to the extraction of mineral resources in absence of recycling, that is $\forall t, m_{t}>0$ if $\delta=0$. In this special case the economy relies on the use of two non-renewable resources as imperfect substitutes for consumption. Along the optimal path, the input ratio is held constant and equal to the relative resource endowment, i.e. $x_{t} / k_{t}=f_{t} / m_{t}=F / M$. The extraction of the two non renewable resources, as well as the production of renewable energy and consumption, decline at the common pace dictated by the factor $(1+\rho)^{-\frac{t}{\varepsilon}} \cdot 18$

When instead the equipment for the production of renewable energy is recyclable, i.e. if $\delta>0$, the argument does not apply to the extraction of minerals. In fact, the production of renewable energy could be positive, i.e. $y_{t}>0$, at some date $t$ even in the absence of contemporaneous extraction of primary mineral resource, i.e. even if $m_{t}=0$, to the extent that the specialized capital stock was positive in the previous period, $K_{t-1}>0$, and it would be precisely equal to $y_{t}=\delta K_{t-1}>0$.

There are two distinct potential reasons for shutting down the mine at some finite date. First of all, the opportunity to recycle minerals embedded in capital introduces an incentive to put forward the extraction date. To see this, consider the extreme case of a $100 \%$ recycling rate, i.e. $\delta=1$. In this case, given our assumption of costless extraction, there is no gain from leaving any mineral resource underground for future use. It is clearly optimal to choose $m_{0}=M$ and $m_{t}=0$ for any $t \geq 1$. In our analysis we take into account the possibility that along the optimal path extraction comes to an end in finite time, and denote by $\bar{t}$ the last period during which extraction is positive. Second, there may be situations where it is preferable to initially keep mines closed and begin extracting only at some later date. This is the case when the economy is endowed of a large initial green capital stock, but only a relatively small stock of primary mineral resources. By choosing $m_{t}=0$ over an initial interval $[0, \underline{t})$, one can delay the use of the limited resource stock $M$ to periods $t \geq \underline{t}$, while keeping the renewable energy input for consumption at rate $y_{t}=K_{-1} \delta^{t+1}$ for $t<\underline{t}$.

We therefore search for the extraction paths of the two resources, such that $\forall t f_{t}>0, \forall t \in[\underline{t}, \bar{t}]$

\footnotetext{
${ }^{18}$ This sub-case is embedded in Proposition 1.
} 
$m_{t}>0$ and otherwise $m_{t}=0$, where periods $\underline{t}$ and $\bar{t}$ have to be chosen. ${ }^{19}$ The planner's problem is

$$
\begin{aligned}
\max _{f_{t}, m_{t}} & \sum_{t=0}^{\underline{t}-1}\left(\frac{1}{1+\rho}\right)^{t}\left(\frac{f_{t}^{\alpha}\left(\delta^{t+1} K_{-1}\right)^{1-\alpha}}{1-\varepsilon}\right)^{1-\varepsilon} \\
& \left.+\sum_{t=\underline{t}}\left(\frac{1}{1+\rho}\right)^{t}\left(\frac{\left.f_{t}^{\alpha}\left(\delta^{t+1} K_{-1}+\sum_{\tau=\underline{t}}^{t} \delta^{t-\tau} m_{\tau}\right)^{1-\alpha}\right)^{1-\varepsilon}}{1-\varepsilon}\right)^{1-\varepsilon}\right)^{1-\varepsilon} \\
& \left.+\sum_{t=\bar{t}+1}^{\infty}\left(\frac{1}{1+\rho}\right)^{t}\left(\frac{f_{t}^{\alpha}\left(\delta^{t+1} K_{-1}+\delta^{t-\bar{t}} \sum_{\tau=\underline{t}}^{\bar{t}} \delta^{\bar{t}-\tau} m_{\tau}\right)^{1-\alpha}}{1-\varepsilon}\right)^{1-\varepsilon}{ }^{\bar{t}}\right) \\
& +\lambda\left(F-\sum_{t=0}^{\infty} f_{t}\right)+\nu\left(M-\sum_{\tau=\underline{t}} m_{\tau}\right)
\end{aligned}
$$

where $\lambda, \nu \geq 0$ are the values of the fossil and mineral resource stocks respectively.

The optimal policy is characterized by the following.

Proposition 1. The unique trajectories solving problem (3.3), are of three types depending on initial capital and resource stocks, and on preference and technological parameters.

1. If the technological efficiency of recycling is above the modified social discount factor $r$, i.e. if $\delta \geq r:=(1+\rho)^{-\frac{1}{\varepsilon}}$, the mineral resource is exhausted in the first period, i.e. $\underline{t}=\bar{t}=0$, while the fossil resource is extracted at an exponentially declining rate

$$
f_{t}=F(1-R) R^{t}
$$

where $R:=\left(\frac{\delta^{(1-\alpha)(1-\varepsilon)}}{1+\rho}\right)^{\frac{1}{1-\alpha(1-\varepsilon)}}$, for all $t \geq 0$.

2. If instead $\delta<r$, both mineral and fossil resources are exhausted over the infinite horizon. There are two distinct types of trajectories in this case.

(a) If the stock of primary mineral resources is abundant relatively to the stock of green capital available in the first period, i.e. if $\frac{M}{\delta K_{-1}} \geq \frac{r-\delta}{1-r}$, both fossil and mineral resources are extracted at all periods, i.e. $\underline{t}=0$ and $\bar{t}=\infty$, and from the second period onward their extraction falls at a common exponential rate, dictated by the modified discount factor, $r$. While fossil resource extraction declines from the first to the second period according to factor $r$, the extraction of the mineral resource between the first and second

\footnotetext{
${ }^{19}$ In our deterministic framework, the optimal policy rules out any path with intermittent extraction of minerals. This is demonstrated in Appendix B.1, but intuitively, the Bellman principle of optimality implies that if along the optimal path extraction comes to an end at $\bar{t}$, it is not efficient to open again the mine at some later period $\tilde{t}>\bar{t}$. Suppose in fact that it is optimal to chose $m_{\tilde{t}}>0$. It makes sense to keep $m_{\tilde{t}-1}=0$ at $\tilde{t}-1$ only if the capital stock $K_{\tilde{t}}$ is considered too large given the remaining stocks of resources $F-\sum_{\tau=0}^{\tilde{t}-1} f_{\tau}$ and $M-\sum_{\tau=t}^{\tilde{t}-1} m_{\tau}$. But these stocks are optimal, since they result of the extraction paths $f_{t}$ and $m_{t}$ up to date $\tilde{t}-1$, assumed to be optimal. Hence, $K_{\tilde{t}}$ cannot be considered excessive. This contradiction shows that our premise, according to which it is optimal to chose $m_{\tilde{t}}>0$ when $m_{\tilde{t}-1}=0$ is optimal, is wrong. Mutatis mutandis the argument holds for the interval $[0, \underline{t})$.
} 
period follows $m_{1}=(r-\delta)\left(m_{0}+\delta K_{-1}\right)=(r-\delta) K_{0}$. The optimal extraction path is

$$
\begin{aligned}
\forall t \geq 0, \quad f_{t} & =(1-r) F r^{t} \\
t=0, \quad m_{0} & =(1-r) \frac{M}{1-\delta}\left(1-\frac{r-\delta}{1-r} \frac{\delta K_{-1}}{M}\right) \\
\forall t>0, \quad m_{t} & =(1-r) \frac{M}{1-\delta}\left(1+\delta \frac{K_{-1}}{M}\right)\left(1-\frac{\delta}{r}\right) r^{t}
\end{aligned}
$$

(b) If instead $\frac{M}{\delta K_{-1}}<\frac{r-\delta}{1-r}$, extraction of the mineral resource is delayed, i.e. $\underline{t} \geq 1$ and $\bar{t}=\infty$. The optimal $\underline{t}$ is the lowest non-negative integer at or above the value $\ln \left(\frac{M}{\delta K_{-1}} \frac{1-r}{r-\delta}\right) / \ln \delta$. Over the first interval of time fossil resource extraction declines according to the factor $R$. From $\underline{t}+1$ onward, the extraction of both resources falls at the common rate $r$. Between period $\underline{t}$ and $\underline{t}+1$ the extraction of fossil resources declines at rate $r$ while that of minerals follows $m_{\underline{t}+1}=(r-\delta)\left(m_{\underline{t}}+\delta^{\underline{t}}+1 K_{-1}\right)$. In this case

$$
\begin{aligned}
\forall t<\underline{t}, \quad m_{t} & =0 \quad ; \quad f_{t}=\left(\frac{1-R^{\underline{t}}}{1-R}+\Gamma(\underline{t}) \frac{r^{\underline{t}}}{1-r}\right)^{-1} F R^{t} \\
\forall t \geq \underline{t}, \quad f_{t} & =\left(\Gamma(\underline{t}) \frac{1-R^{\underline{t}}}{1-R}+\frac{r^{\underline{t}}}{1-r}\right)^{-1} F r^{t} \\
t=\underline{t}, \quad m_{\underline{t}} & =(1-r) \frac{M}{1-\delta}\left(1-\delta^{\underline{t}} \frac{r-\delta}{1-r} \frac{\delta K_{-1}}{M}\right) \\
\forall t>\underline{t}, \quad m_{t} & =\frac{1-r}{1-\delta}\left(M+\delta^{\underline{t}+1} K_{-1}\right)\left(1-\frac{\delta}{r}\right) r^{t-\underline{t}}
\end{aligned}
$$

where $\Gamma(\underline{t}):=\left(\frac{r \delta(1-\delta) K_{-1}}{(1-r)\left(M+\delta^{\underline{t}+1} K_{-1}\right)}\right)^{\frac{(1-\alpha)(1-\varepsilon)}{1-\alpha(1-\varepsilon)}}$.

Proof. The detailed proof is in Appendix A and B.

Let us explain the optimal trajectories of resource extraction and energy production specified in Proposition 1 and comment on them.

First, notice that the Hotelling principle for the efficient management of non renewable resources applies to our framework. When the optimal policy maintains a constant input ratio, consumption falls at the same rate as the common rate driving the decline in resource extraction. Say that $q$ declines at a factor $g \in(0,1)$, i.e. $q_{t+1}=g q_{t}$. Then the value of a marginal unit of the resource mix increases at rate $p_{t+1} / p_{t}=g^{-\varepsilon}$. Along the optimal trajectory from period 1 onward in case (2.a), or from period $\underline{t}+1$ onward in trajectory (2.b), the optimal path of resource extraction implies $g=r$, therefore $p_{t+1} / p_{t}=r^{-\varepsilon}=1+\rho$ : the value of a marginal unit of resource increases at the pure discount rate, as in Hotelling (1931). ${ }^{20}$

Second, the asymmetry between the two types of resources, concerning the possibility to recycle them, implies a difference in their optimal extraction paths. To see this let us focus on the case of

\footnotetext{
${ }^{20} p_{t}$ is the marginal value of energy services, to which the marginal values of mineral and fossil resources extracted are proportional.
} 
moderate recycling $(\delta<r)$ and no endowment of green capital $\left(K_{-1}=0\right)$, a sub-case of (2.a) in Proposition 1. In this case, the initial ratio of resource extraction $f_{0} / m_{0}$ equals the initial input ratio $f_{0} / K_{0}$. As previously argued, without recyclability, it is optimal to choose $f_{0} / m_{0}=F / M$, according to the relative resource endowment (set $K_{-1}=\delta=0$ in (3.6) and compare to (3.5)). When green capital can be recycled, but $K_{-1}=0$, we see from (3.6) that the extraction and input ratios are initially biased toward more intensive use of minerals $f_{0} / m_{0}=(1-\delta) F / M$. This first period choice is the same as the one made in an economy endowed of a larger stock of non renewable and non recyclable mineral resources of size $\widehat{M}:=M /(1-\delta)$. The stock $\widehat{M}$ is the initial endowment adjusted for recycling and measures the maximum feasible amount of mineral inputs that can be used in the production of renewable energy over time, i.e. $\widehat{M}=\sum_{t=0}^{\infty} \delta^{t} M$ obtained by extracting all minerals in the first period $(\underline{t}=0$ and $\bar{t}=0)$. This observation points to the fact that the possibility of recycling the mineral resource embedded in green capital is equivalent to an endowment of a larger stock of mineral resources. Since, due to $\delta>0$, mineral resources are relatively more abundant, the constant input ratio $f_{t} / K_{t}$ is optimally chosen lower. However, the ratio of resource extraction $f_{t} / m_{t}$ can only be kept constant from period 1 onward, if mineral extraction is adjusted at date 0 to account for the absence of recycled resources at that date. In so doing, the input ratio $f_{t} / K_{t}$ remains constant. As a consequence the ratio of resource extraction, $f_{t} / m_{t}$, is increased after the initial period. ${ }^{21}$ The following statement summarizes this analysis.

Corollary 1. When $K_{-1}=0$ and $\delta<r:=(1+\rho)^{-\frac{1}{\varepsilon}}$, the solution of problem (3.3) implies that the larger is the recycling rate $\delta \in[0, r)$, the more intensive in renewable energy is the constant input ratio, the greater is the extraction of minerals in the first period and green capital at every period, the more are extracting activities concentrated on minerals initially and on fossil resources from the second period onward.

$$
\forall t \geq 0 \frac{x_{t}}{y_{t}}=\frac{f_{t}}{K_{t}}=\frac{F}{\widehat{M}} \quad ; \quad m_{0}=(1-r) \widehat{M} \quad ; \quad \forall t \geq 1 \frac{f_{t}}{m_{t}}=\frac{r}{r-\delta} \frac{F}{\widehat{M}}
$$

Proof. The value of $m_{0}$ is an application of (3.6) in Proposition 1. We have $\frac{\partial m_{0}}{\partial \delta}=\frac{m_{0}}{1-\delta}>0, \frac{d K_{t}}{d \delta}=\frac{d m_{0}}{d \delta} r^{t}>0$ and $\frac{d m_{t}}{d \delta}=-\left(\frac{1-r}{1-\delta}\right)^{2} M r^{t}<0$. The result on the input ratio holds because, as argued in the main text $f_{t}$ and $K_{t}$ grow at the same rate $r$ at any date. Applying results for the case (2.a) in Proposition 1, we get the ratio of resource extraction for $t \geq 1$. Thus $\frac{\partial f_{t} / m_{t}}{\partial \delta}=r \frac{1-r}{(r-\delta)^{2}} \frac{F}{M}>0$ and $\frac{f_{0}}{m_{0}}<\frac{f_{t}}{m_{t}}$ for $t \geq 1$.

Figure 2 illustrates the optimal paths of extraction of mineral and fossil resources, of green capital and consumption. ${ }^{22}$ It represents the cases of two economies differing by the recycling rate $\delta$ under the assumption of case (2.a) where $r>\delta$ and no green capital endowment. According to (3.5) fossil extraction does not depend on $\delta$. We can see that the dynamics of mineral resource extraction $m$ is qualitatively affected (the two curves cross each other), while that of consumption $q$ and green capital $K$ only shift upwards in levels with the rate of recycling.

\footnotetext{
${ }^{21} K_{-1}=0$ implies $f_{0} / K_{0}=f_{0} / m_{0}$. From case (2.a) in Proposition $1 \forall t \geq 1, f_{t} / m_{t}=f_{t+1} / m_{t+1}$, and $f_{1} / m_{1}=$ $r f_{0} /\left((r-\delta) K_{0}\right)$. Hence the upward jump in the extraction ratio from period 0 to period $1: f_{1} / m_{1}>f_{0} / m_{0}$.

${ }^{22}$ Our benchmark calibration is $\rho=.04, \delta=.5, \alpha=.7, K_{-1}=0, M=.5, F=3, \frac{q}{f^{\alpha} K^{1-\alpha}}=4$. We choose $\varepsilon=.2$ for an illustrative purpose, since most of the dynamics takes place over the very first periods in this case.
} 


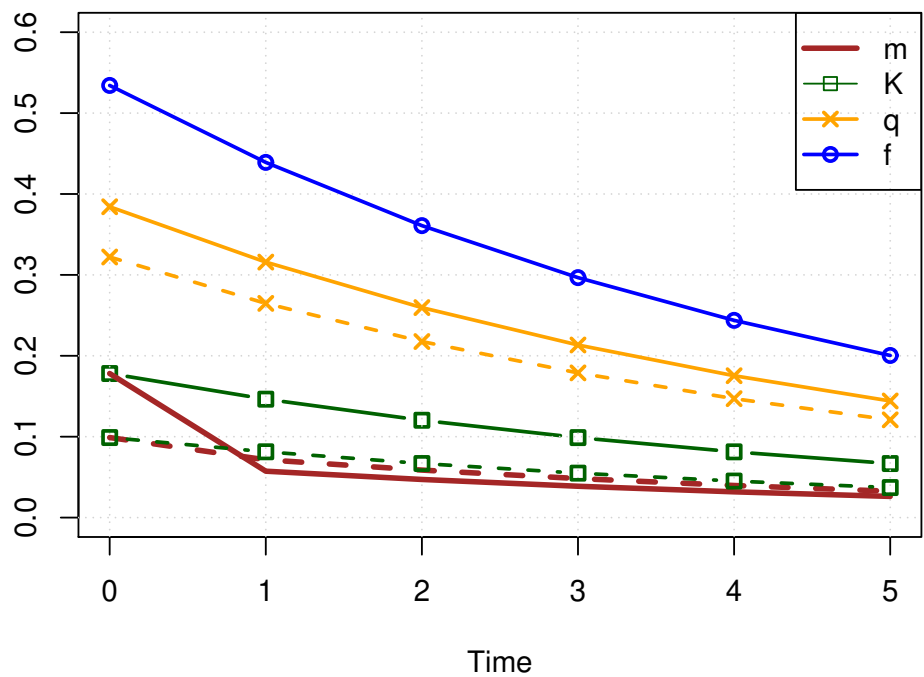

Figure 2: Resource extraction, green capital and consumption paths for two different recycling rates: continuous lines for $\delta=0.5$, dashed lines for $\delta=0.1$ (see calibration footnote 22).

The results in Corollary 1 have relevant policy implications. On the one hand, the empirically grounded observation that the production of renewable energy relies on the use of specific non renewable resources, namely minerals, suggests that the economy is poorer than it would be if the renewable energy could be produced out of non exhaustible inputs. From this point of view, the observation points to a limitation of renewable energy as a factor to overcome the limits to growth. In terms of our framework, this argument is represented by the lower value of welfare ceteris paribus when $\delta<1$ than when $\delta=1 .^{23}$ This observation provides an argument stating that the potential production of renewable energy is more limited than generally thought. We refer to this argument as the pessimistic stance.

On the other hand, our analysis illustrates that the possibility to recycle minerals embedded in green capital makes it preferable to choose an energy mix composed of more renewable energy and less conventional fossil resources. Hence, adding a plausible assumption on the recycling technology to the same empirical observation, we provide a pro renewable energy argument, partially countering the pessimistic stance.

Moreover, we present an original argument in favor of a pro active renewable energy policy. We show that for a given amount of mineral resources to be devoted to the production of renewable energy, we should skew extraction toward the present the greater the recyclability of minerals. In other words, because minerals are recyclable and fossil resources are not, we should develop as soon as possible the green capital embedding the minerals, that allows us to produce renewable energy and to substitute for conventional fossil energy. This is found in Corollary 1, as well as in the extreme in case (1.) of Proposition 1 where minerals are entirely embedded in green capital from

\footnotetext{
${ }^{23}$ In fact, welfare always increases with $\delta$ (online Appendix $\mathrm{H}$ ).
} 


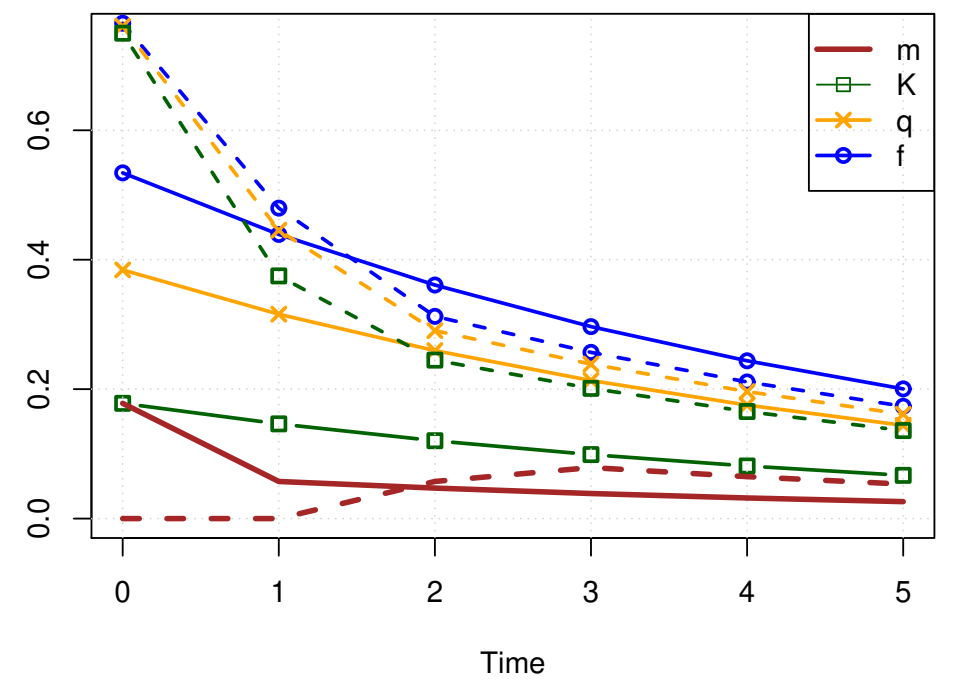

Figure 3: Resource extraction, green capital and consumption paths for two endowments in green capital: continuous lines for $K_{-1}=0$, dashed lines for $K_{-1}=1.5$ (see calibration footnote 22).

$t=0$. Notice that this original pro active argument is grounded on the same empirical observation underlying the pessimistic stance. It relies on the flexibility in scheduling resource use typical of the management of non renewable resources. In fact, putting forward the potential of future production of renewable energy is off the production possibility set in commonly used models with renewable and non renewable sources of energy (e.g. Moreaux and Ricci, 2005).

Our discussion above abstracts from several potential reasons for putting forward or for postponing investment in green capital. In the next section we review a few of them. The framework in Proposition 1 provides already a possible reason for delaying investment. It could be that at the start of the planning horizon, the economy has inherited of a large stock of green capital. If previous investment decisions were not optimal, and inefficiently biased toward renewable resources, the resulting stock of green capital, and thus of secondary mineral resource available in the first period, could exceed the desirable initial stock of capital for the first period. This corresponds to case (2.b) in Proposition 1. Figure 3 shows how the optimal paths of four endogenous variables -extraction of fossil and mineral resources, green capital, and consumption- vary with the endowment of green capital. The level of this endowment is chosen to represent the qualitative features of cases (2.a) and (2.b) in Proposition 1. In the latter case it is optimal to delay the extraction of mineral resources (as $\underline{t}>0$ ). Nevertheless, in this case, production of renewable energy is initially quite high, and actually higher than socially desirable. In practice, this case may be of little relevance.

Two further remarks on case (2.b) are worthwhile. First, over the interval of time $[0, \underline{t})$ the stock of green capital declines at rate $\delta$ instead of the socially desired rate $r>\delta$. Though abundant, green capital is still valuable because productive, and it is therefore used at full capacity. As a result though, over this interval of time, the rate at which fossil extraction decreases is adjusted 
and differs from the one prevailing in presence of mineral extraction. ${ }^{24} \mathrm{~A}$ similar adjustment to the extraction of fossil resources applies in case (1.) of a sufficiently efficient recycling technology. Second, the marginal effect of an increase in the recycling rate $\delta$ is more complex in the case (2.b) of Proposition 1 than in the case (2.a) treated in Proposition 1. On the one hand the same forces presented in Corollary 1 apply. Yet now, a countervailing effect operates through the fact that more secondary mineral resources are made available by the increase in $\delta$ over the initial interval $[0, \underline{t})$. The beginning of extraction may be delayed, and the initial extraction of mineral resources may decline with $\delta .^{25}$ That being said, in general, the higher the recycling rate, the higher the optimal initial extraction of minerals, and the lesser the subsequent ones, unless reserves $M$ are very low as compared to endowment in green capital. Indeed, the higher the recycling rate, the more abundant are resources in the future. This weakens the trade-off between present and future consumption, allowing for earlier extraction. However, absent sufficient reserves for the future, another effect dominates: the higher the recycling rate, the more one benefits from past investment in green capital, and the less one needs to extract minerals in the future. In practice, we argue that recoverable resources $M$ is one order of magnitude larger than green capital $K_{-1}$ for base metals (Singer, 2017), so we can reasonably assume that the realistic case is the case (2.a), ${ }^{26}$ with $K_{-1} \approx 0$ and $\underline{t}=0$.

\section{Extensions in a two-period model}

In the previous section, we analyzed how the asymmetry in recyclability between inputs used in the production of conventional vs. renewable energy affects the optimal timing of energy production. In this section, we consider other factors affecting this timing, and in particular initial investment in green capital $m_{0}$ and the energy mix. In order to develop these extensions in a clear and tractable way, we consider the two-period version of the model presented in section 2 , with $t \in\{0 ; 1\}$. We check the validity of the following results in a number of extensions. First, the existence of a threshold on recyclability of minerals such that primary mineral resources are exhausted in the first period, for $\delta$ above the threshold, as established in Proposition 1. Second, the fact that, for $\delta$ below this threshold, a marginal increase in recyclability fosters first period mineral resources use and investment in green capital (i.e. $\partial m_{0} / \partial \delta, \partial K_{0} / \partial \delta>0$ ), and makes the input ratio more intensive in renewable energy $\left(\partial\left(f_{0} / K_{0}\right) / \partial \delta<0\right)$, as established in Corollary $1 .^{27}$

\footnotetext{
${ }^{24}$ More precisely, we deduce from Appendix $\mathrm{C}$ that the optimal rate of decay for fossil extraction is the closest one between $r$ and $R$ from the pure discount factor $\frac{1}{1+\rho}$.

${ }^{25}$ We have $\frac{d m_{\underline{t}}}{d \delta}>0 \Longleftrightarrow \forall t>\underline{t}, \frac{d m_{t}}{d \delta}<0 \Longleftrightarrow M>\delta^{\underline{t}} K_{-1}\left(\frac{(r-\delta)(1-\delta)}{1-r}(\underline{t}+1)-\delta\right)$ and $\frac{d \underline{t}}{d \delta}=\frac{1}{(r-\delta) \ln (\delta)}-$ $\ln \left(\frac{M}{K_{-1}} \frac{1-r}{r-\delta}\right) / \delta \ln ^{2}(\delta)$. One can check that, even in the range of parameters of case $(2 . b)\left(\delta<r\right.$ and $\left.\frac{M}{\delta K_{-1}}<\frac{r-\delta}{1-r}\right)$ both signs are possible for each of these derivatives.

${ }^{26}$ Indeed, $\delta \geq r$ does not seem realistic. An upper credible value for the pure discount rate $\rho$ is 0.05 , while the inverse of the elasticity of intertemporal substitution $\varepsilon$ can reasonably be assumed higher than 0.5. Combining these conservative figures gives a low estimate for $r$ : 0.9 . Taking more common values for $\rho$ and $\varepsilon$ would yield an even higher threshold $r$, so that for any realistic value of the recycling rate $\delta$, it is extremely likely to have $\delta<r$ and to be in the case where the optimal path is an endless extraction.

${ }^{27}$ These are the main original and policy relevant results of our analysis. In the versions of model presented in this section, second period outcomes are affected by the fact that it is the last period in a finite horizon with non renewable
} 
To begin with, we show how the results adjust to the finite horizon case, studying the benchmark case with constant relative risk aversion (CRRA) utility function. We disentangle two mechanisms by first studying the sub-case of a logarithmic utility, then discuss the role of the preference for intertemporal consumption smoothing in the optimal timing of energy production. Within this simplified framework, we study how expected technological progress, such as improved productivity of minerals in renewable energy equipment, affects the optimal investment in green capital. We move on to consider alternative assumptions on the production technology concerning the degree of substitutability between energy services provided by the two types of resources within each period. ${ }^{28}$ Next, we consider the role played by convex extraction costs in determining the optimal time of investment in green capital. Finally, we allow for environmental damages from the use of fossil resources, which also affect the optimal path of resource use.

\subsection{The benchmark model with technological change}

Let us consider first the case with CRRA utility function, Cobb-Douglas production function and non constant productivities of resource inputs. In a two-period setting the planner's problem is as follows:

$$
\begin{gathered}
\max \frac{q_{0}^{1-\varepsilon}}{1-\varepsilon}+\frac{1}{1+\rho} \frac{q_{1}^{1-\varepsilon}}{1-\varepsilon} \\
q_{t}=\left(A_{t} f_{t}\right)^{\alpha}\left(B_{t} K_{t}\right)^{1-\alpha}, t \in\{0 ; 1\} \\
K_{0}=m_{0}, K_{1}=m_{1}+\delta m_{0} \\
f_{0}+f_{1} \leq F \\
m_{0}+m_{1} \leq M
\end{gathered}
$$

with $m_{0}, m_{1}, f_{0}$, and $f_{1} \geq 0$, where $\alpha \in(0,1)$ and $\varepsilon>0$ (log utility for $\left.\varepsilon=1\right)$.

Proposition 2. The unique trajectories solving problem (4.1) are of two types. If $\delta<\widetilde{\delta} \equiv \frac{\widetilde{r}}{1+\widetilde{r}}$ where

$$
\widetilde{r}:=\frac{1}{1+\rho}\left(\frac{q_{1}}{q_{0}}\right)^{1-\varepsilon}
$$

it is optimal to extract the mineral resource in both periods, as follows

$$
m_{0}=\frac{\frac{1}{1-\delta}}{1+\widetilde{r}} M ; m_{1}=\frac{\widetilde{r}-\frac{\delta}{1-\delta}}{1+\widetilde{r}} M ; f_{0}=\frac{1}{1+\widetilde{r}} F ; f_{1}=\frac{\widetilde{r}}{1+\widetilde{r}} F
$$

implying

$$
\frac{q_{1}}{q_{0}}=\left[\left(\frac{A_{1}}{A_{0}}\right)^{\alpha}\left(\frac{B_{1}}{B_{0}}\right)^{1-\alpha}\right]^{\frac{1}{\varepsilon}}(1+\rho)^{-\frac{1}{\varepsilon}}(1-\delta)^{\frac{1-\alpha}{\varepsilon}}
$$

resources. Hence the results we obtain for second period outcomes are less interesting and robust, that those for $m_{0}$ and $f_{0} / K_{0}$ on which we focus.

${ }^{28}$ Alternatively, this can be interpreted as a feature related to preferences. 
Moreover $\frac{f_{0}}{K_{0}}=\frac{F}{\widehat{M}}, \frac{f_{1}}{K_{1}}=\frac{F}{M}$ and $\frac{K_{1}}{K_{0}}=\widetilde{r}(1-\delta)$. Therefore $\frac{\partial m_{0}}{\partial \delta}>0, \frac{\partial f_{0} / K_{0}}{\partial \delta}<0, \frac{\partial f_{1} / K_{1}}{\partial \delta}=0$, $\frac{d\left(q_{1} / q_{0}\right)}{d \delta}<0$ and $\frac{\partial K_{1} / K_{0}}{\partial \delta}<0$.

If $\delta \geq \widetilde{\delta}$, where $\tilde{\delta}$ is defined using (4.2) and (4.4), the mineral resource is exhausted at date 0. The optimal resource use is $m_{0}=M, m_{1}=0, f_{0}=\frac{1}{1+\zeta} F$, and $f_{1}=\frac{\zeta}{1+\zeta} F$, where $\zeta:=$ $\left(\left(\frac{A_{1}}{A_{0}}\right)^{\alpha}\left(\frac{B_{1}}{B_{0}}\right)^{1-\alpha} \frac{1}{1+\rho} \delta^{(1-\alpha)(1-\varepsilon)}\right)^{\frac{1}{1-\alpha(1-\varepsilon)}}$. In this case $\frac{q_{1}}{q_{0}}=\left(\left(\frac{A_{1}}{A_{0}}\right)^{\alpha}\left(\frac{B_{1}}{B_{0}}\right)^{1-\alpha} \frac{1}{1+\rho}\right)^{\frac{-\alpha}{1-\alpha(1-\varepsilon)}} \delta^{\frac{1-\alpha}{1-\alpha(1-\varepsilon)}}$ instead of (4.4). Hence $\frac{d\left(q_{1} / q_{0}\right)}{d \delta}>0$, while $\frac{d f_{0}}{d \delta}<0, \frac{d f_{1}}{d \delta}>0$ if $\varepsilon<1$ but $\frac{d f_{0}}{d \delta}>0$, $\frac{d f_{1}}{d \delta}<0$ if $\varepsilon>1$.

Proof. See Appendix D.

From (4.2) and (4.4), $\tilde{r}$ is the ratio of the present value current utility from energy consumption when $\delta<\widetilde{\delta}$. Hereafter, we refer to this ratio as the gross social discount factor. Let us begin by considering the sub-case without technological progress $\left(A_{1} / A_{0}=B_{1} / B_{0}=1\right)$. Moreover, first consider the case of logarithmic utility $(\varepsilon=1)$. In this case, the threshold value of the recycling rate, $\tilde{\delta}=\frac{1}{2+\rho}$, is independent of the recycling rate and relative resource abundance, since $\tilde{r}$ is equal to the pure discount factor $\frac{1}{1+\rho}$ (see $\left.(4.2)\right)$. When $\delta>\widetilde{\delta}$, all mineral resources are extracted and used in the first period, i.e. $m_{0}=M$, and the decline in energy consumption is given by $\frac{q_{1}}{q_{0}}=\left(\frac{1}{1+\rho}\right)^{\alpha} \delta^{1-\alpha}$ and increases with $\delta$. Otherwise, for $\delta$ below the threshold, minerals are extracted in both periods and consumption of energy services declines at $\frac{q_{1}}{q_{0}}=\frac{1}{1+\rho}(1-\delta)^{1-\alpha}$, a decreasing function of $\delta$. In this case, the higher the rate of recycling, the earlier the use of primary mineral resources, the larger the initial investment in green capital, and the more intensive in renewable energy is the input ratio in the first period. These three results confirm those in Proposition 1 and Corollary 1.

Other results differ from the case with infinite horizon. First, the input ratio in the second period does not change with the rate of recycling. This difference is not surprising, since there is no advantage from recycling mineral resources used in the second period in a setting where there is no future period to use recycled resources (i.e. no third period). Second, as noticed, when $\delta<\tilde{\delta}$, energy consumption declines at a faster pace the greater is the recycling rate, while this rate of decline is unaffected by $\delta$ in the infinite horizon case. Improved $\delta$ tends to increase $q_{1} / q_{0}$ for unchanged intertemporal resource allocation. Yet, it also makes it more interesting to extract minerals in the first period, reducing $q_{1} / q_{0}$. This second substitution effect dominates the former when $\delta<\tilde{\delta}$. Finally, a higher $\delta$ affects $q_{1} / q_{0}$ through the positive income effect that calls for an intertemporal reallocation of mineral and fossil resources, according to social preferences on consumption smoothing, to which we turn our attention below. Notice that in this case with logarithmic utility and Cobb-Douglas production functions, the opportunity to recycle mineral resources does not affect the use of fossil resources.

As a second step, consider the case $\varepsilon \neq 1$ to study the role of preferences with respect to intertemporal consumption smoothing, in determining the timing of resource use and investment in green capital. We find that, when $\delta<\tilde{\delta}$, the decline in energy consumption (4.4) is a decreasing function of $\delta$, more so the smaller is $\varepsilon$, i.e. the greater the elasticity of intertemporal substitution, $1 / \varepsilon$. An improvement in the recyclability of minerals brings forward mineral resource use more so the least adverse to variability in the consumption over time is the representative household, 
and as a consequence the larger is the downward adjustment in optimal consumption. Since $\tilde{r}=$ $(1+\rho)^{-\frac{1}{\varepsilon}}(1-\delta)^{\frac{1-\varepsilon}{\varepsilon}(1-\alpha)}$ (from (4.2) and (4.4)), the gross discount rate is affected by $\varepsilon$ through two channels. First, the pure preference for the present, $\rho$, which directly affects the gross discount factor and therefore the timing of consumption and thus of resource use. Second, the gross discount factor is affected by the prospective decline in consumption, given by (4.4), itself influenced by the recycling technology for green capital. The expected decline in consumption tends to decrease the gross discount factor if $\varepsilon$ is smaller than unity, i.e. for high elasticity of intertemporal substitution of consumption $1 / \varepsilon$, and vice versa. As a result, the resource use tends to be brought forward, thus $f_{1} / f_{0}$ and $m_{1} / m_{0}$ to decrease. It is worthwhile noticing the asymmetry between the two resources. Inspecting (4.3) we see that a marginal increase in $\delta$ exerts two effects on $m_{0}$, a direct one and an indirect one through $\tilde{r}$. As established in Proposition 2, the former force dominates, so that an increase in $\delta$ reduces the ratio $m_{1} / m_{0}$, bringing forward mineral resource use and boosting investment in green capital during the first period, whatever $\varepsilon$. Nevertheless, the impact is smaller the larger is the willingness to smooth consumption over time if $\varepsilon>1$, and vice versa, because of the above mentioned increase in the gross discount factor. In the case of fossil resources instead, only this indirect effect running through the gross discount factor is at work, so that the optimal fossil resource use is delayed ( $f_{1} / f_{0}$ increases) if $\varepsilon>1$ but it is brought forward (declines) if $\varepsilon<1$. If we interpret the objective of the planner as a welfare function across two generations, the parameter $\varepsilon$ determines inequality aversion. While an increase in $\delta$ implies a sharper decrease of consumption across generations, the size of this change is milder the higher inequality aversion.

Our analysis shows that preferences with respect to intertemporal substitution in consumption possibilities play an important role in determining the optimal timing of resource use and investment in green capital. However, the original mechanism underscored in this paper, based on the asymmetry between the two types of resources, is still crucially at work in determining the optimal timing of investment in green capital, making it preferable to bring forward investment as the efficiency of the recycling technology increases.

Finally, consider the effect of expected technological change. The asymmetry on the optimal timing of resource use implied by the possibility to recycle minerals embedded in green capital is unaffected, since the results concerning the role of parameter $\delta$ hold independently of $B_{1} / B_{0}$. Nevertheless, in the case of an interior solution $(\delta<\tilde{\delta})$ prospects of technological progress do affect the optimal timing in resource use and investment in green capital, through their influence on the optimal rate of growth of energy consumption (4.4). Expected improvements in the productivity of green capital, i.e. $B_{1}>B_{0}$, lead to higher consumption growth $\partial\left(q_{1} / q_{0}\right) / \partial B_{1}>0$. This, in turn, exerts wider effects, according to the attitude toward consumption smoothing. If $\varepsilon<1$, slower decline in energy consumption increases the gross social discount factor $\partial \widetilde{r} / \partial B_{1}>0$, and therefore delays the extraction of mineral $\partial m_{0} / \partial B_{1}<0$ and fossil $\partial f_{0} / \partial B_{1}<0$ resources, while raising the threshold value on recyclability of minerals $\partial \widetilde{\delta} / \partial B_{1}>0$. The opposite consequences apply if $\varepsilon>1$.

In the analysis hereafter we abstract again from technological change and assume again $\forall t$ $A_{t}=B_{t}=1$. 


\subsection{Substitutability between energy services from different sources}

Until now, we have assumed the specific Cobb-Douglas form (3.1) for the production function of energy services (2.2) combining services from fossil and renewable energy. This assumption simplifies the analysis, but there is no reason to believe that these two types of energy services are substitutes among each other with a constant and unitary elasticity of substitution. Thinking of electricity as an homogeneous good, one might consider that the elasticity of substitution is much larger than unity. Alternatively, one might view renewable and conventional sources of energy as quite imperfect substitutes in providing energy services, due to the intermittent availability of some renewable sources of electricity, or to physical properties (weight, density, caloric power) of some fossil sources of energy, making them drastically more efficient in some uses other than electricity production (e.g. air transportation).

The degree of substitutability between the two types of energy services may affect the optimal timing of investment in green capital. To see why, consider the heuristic extreme case without recycling, nor discounting, and completely inelastic preferences over the intertemporal consumption path of energy services $(\varepsilon=\infty)$. The objective is maximized by keeping constant at $q_{t}=Q\left(\frac{1}{2} F, \frac{1}{2} M\right)$, whatever the elasticity of substitution between the arguments in function $Q($.$) . If this elasticity$ is nil, it is optimal to use half of each resource per period. If the elasticity of substitution is very large, then there is a continuum of combinations of fossil and minerals (green capital) that maximize welfare, and therefore some minerals can be used in the first period to build up more green capital, $m_{0}>\frac{1}{2} M$ (though leaving welfare unaffected). Introducing recycling of green capital into the picture, the latter feature changes: welfare may be increased by bringing forward investment in green capital. In doing so, the secondary resource stock of minerals increases, so that renewable energy services in the second period decrease by less than their increment in the first period. This potentially beneficial role of recycling is less valuable in the case of moderate possibilities for substituting between the two types of energy services. In the limit, if the latter are perfect complements, bringing forward mineral extraction does not create additional value and the optimal resource use is unaffected by $\delta$. This discussion suggests that the elasticity of substitution between the two types of energy services interacts with the preference parameters, namely the elasticity of intertemporal substitution of energy consumption, in determining the optimal timing of investment in green capital.

Modifying the planner's problem (4.1), by substituting $q_{t}=\left(\alpha f_{t}^{\frac{\sigma-1}{\sigma}}+(1-\alpha) K_{t}^{\frac{\sigma-1}{\sigma}}\right)^{\frac{\sigma}{\sigma-1}}$ for $q_{t}=\left(A_{t} f_{t}\right)^{\alpha}\left(B_{t} K_{t}\right)^{1-\alpha}$ with $\sigma>0$, we find that the interior solution (i.e. $m_{1}>0$ ) holds if the recycling rate is below a threshold, i.e. if $\delta<\frac{\widetilde{r}}{(1-\delta)^{1-\sigma}+\widetilde{r}}$, where we extend the definition of $\tilde{r}$ as follows

$$
\widetilde{r}:=\left(\frac{1}{1+\rho}\right)^{\sigma}\left(\frac{q_{1}}{q_{0}}\right)^{1-\varepsilon \sigma}
$$

In this case, efficient resource extraction is defined by (4.3) but for the following 29

\footnotetext{
${ }^{29}$ See Appendix E for the derivation of the results presented in this sub-section.
} 


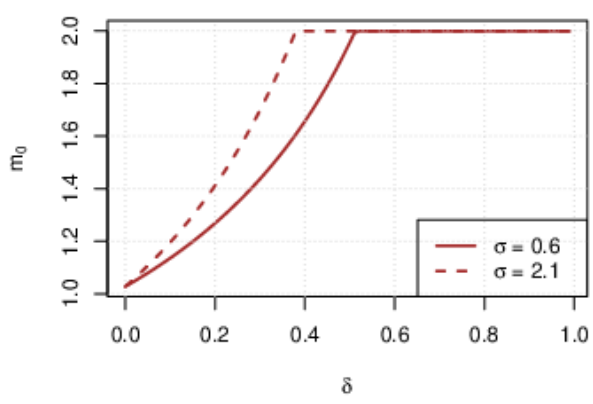

(a) Initial investment in green capital

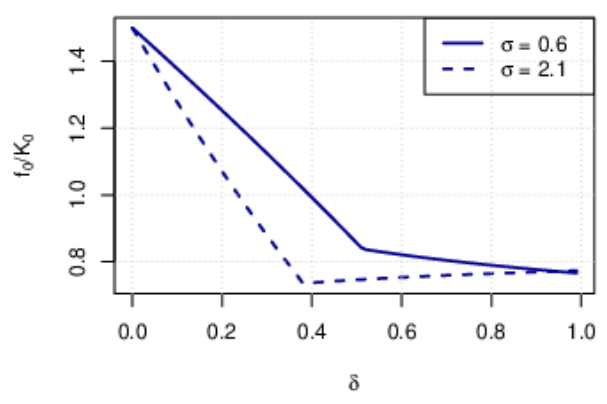

(b) First period energy mix

Figure 4: How the solution varies with the efficiency of the recycling technology in the case of a CES production function for energy services (see calibration footnote 22).

$$
m_{0}=\frac{1}{1+\widetilde{r}(1-\delta)^{-(1-\sigma)}} \widehat{M} \quad, \quad m_{1}=\frac{\widetilde{r}(1-\delta)^{\sigma}-\delta}{1+\widetilde{r}(1-\delta)^{-(1-\sigma)}} \widehat{M}
$$

and the intertemporal energy consumption ratio $\frac{q_{1}}{q_{0}}$ is implicitly defined as the solution of

$$
G\left(\frac{q_{1}}{q_{0}}, \delta\right)-\left(\frac{q_{1}}{q_{0}}\right)^{\varepsilon \sigma}(1+\rho)^{\sigma}=0
$$

where

$$
G\left(\frac{q_{1}}{q_{0}}, \delta\right) \equiv\left(\frac{\alpha F^{\frac{\sigma-1}{\sigma}}+(1-\delta)^{\sigma-1}(1-\alpha) M^{\frac{\sigma-1}{\sigma}}\left(\frac{1+\left(\frac{q_{1}}{q_{0}}\right)^{1-\varepsilon \sigma}(1+\rho)^{-\sigma}}{1-\delta+\left(\frac{q_{1}}{q_{0}}\right)^{1-\varepsilon \sigma}(1+\rho)^{-\sigma}(1-\delta)^{\sigma}}\right)^{\frac{\sigma-1}{\sigma}}}{\alpha F^{\frac{\sigma-1}{\sigma}}+(1-\alpha) M^{\frac{\sigma-1}{\sigma}}\left(\frac{1+\left(\frac{q_{1}}{q_{0}}\right)^{1-\varepsilon \sigma}(1+\rho)^{-\sigma}}{1-\delta+\left(\frac{q_{1}}{q_{0}}\right)^{1-\varepsilon \sigma}(1+\rho)^{-\sigma}(1-\delta)^{\sigma}}\right)^{\frac{\sigma-1}{\sigma}}}\right)^{\frac{\sigma}{\sigma-1}}
$$

As shown in Figure 4, the numerical solutions confirm that $\frac{\partial m_{0}}{\partial \delta}>0$ and $\delta<\widetilde{\delta} \Longrightarrow \frac{\partial f_{0} / K_{0}}{\partial \delta}<0$, hence that our argument applies also in this case. ${ }^{30}$ The solution changes with $\sigma$ : the marginal impact of $\delta$ on the initial investment in green capital and energy mix is stronger as the elasticity of substitution between energy services increases. Moreover, this dependency is positively related to the elasticity of intertemporal substitution, $1 / \varepsilon$. These findings confirm the heuristic argument developed in the previous paragraph: the flexibility in combining the two types of energy services affects the optimal timing of green investment and the optimal energy mix when green capital can be recycled. Specifically the more flexible are the preferences and the technology, the more society takes advantage of the opportunity opened by recycling. Yet, our original argument favorable to early investment and to the intensity in renewables of the energy mix apply in this more involved

\footnotetext{
${ }^{30}$ The sensitivity analysis confirms that these results are robust. In particular, they hold for all combinations of $\sigma \in[0.1,10.1]$ and $\varepsilon \in[0.1,6.1]$. The code used for this and the other numerical results, including the Figures, is available on-line with the supplementary material.
} 
setting. To the extent that much of the relative mineral intensity of renewables is linked to the electricity-storage technology and the aim of the latter is precisely to improve the substitutability between conventional and renewable sources of electricity, our argument is somewhat reinforced by the analysis, though of course it assumes both parameters as exogenous.

\subsection{Convex extraction costs}

So far, in our analysis we ruled out extraction costs. If the marginal costs of producing fossil and mineral resources increase with the extraction rate, the optimal timing of resource use, thus of investment in green capital, should be affected. In order to show this in a clear-cut way, we present a variant of our two-period model where fossil resources play no role. ${ }^{31}$ We focus on the optimal solution with full exhaustion of the minerals stock and $m_{1}>0,{ }^{32}$ and consider the following planner's problem:

$$
\max \frac{1}{1-\varepsilon} m_{0}^{1-\varepsilon}+\frac{1}{1+\rho} \frac{1}{1-\varepsilon}\left(M-(1-\delta) m_{0}\right)^{1-\varepsilon}-\frac{c}{1+\gamma} m_{0}^{1+\gamma}-\frac{1}{1+\rho} \frac{c}{1+\gamma}\left(M-m_{0}\right)^{1+\gamma}
$$

where $c, \gamma>0$ are the extraction cost parameters, and minerals are exhausted $m_{1}=M-m_{0}$. The first order condition is

$$
P\left(m_{0}\right):=m_{0}^{-\varepsilon}-\frac{1-\delta}{1+\rho}\left(M-(1-\delta) m_{0}\right)^{-\varepsilon}=c\left(m_{0}^{\gamma}-\frac{1}{1+\rho}\left(M-m_{0}\right)^{\gamma}\right)=: C\left(m_{0}\right)
$$

$P\left(m_{0}\right)$ measures the present value of the marginal utility generated by first period mineral extraction. It is a monotonically decreasing function of $m_{0}$, taking values $+\infty$ for $m_{0}=0$, and $M^{-\varepsilon}\left(1-\frac{(1-\delta)}{1+\rho} \delta^{-\varepsilon}\right)$ for $m_{0}=M$. It becomes nil at $m_{0}^{u}:=\left(1-\delta+\left(\frac{1-\delta}{1+\rho}\right)^{\frac{1}{\varepsilon}}\right)^{-1} M . C\left(m_{0}\right)$ measures the present value of the marginal cost of extraction at date 0 . It is an increasing function of $m_{0}$, from $-c \frac{1}{1+\rho} M^{\gamma}$ for $m_{0}=0$ up to $c M^{\gamma}$ for $m_{0}=M$. It becomes nil at $m_{0}^{c}:=$ $\left(\frac{1}{1+\rho}\right)^{\frac{1}{\gamma}}\left(1+\left(\frac{1}{1+\rho}\right)^{\frac{1}{\gamma}}\right)^{-1} M$. Therefore, if the two schedules cross in the space $\left(m_{0}\right.$, value) for $m_{0} \in[0, M]$, they do so only once. We conclude that, if $\left(1-\frac{1-\delta}{1+\rho} \delta^{-\varepsilon}\right)<c M^{\gamma+\varepsilon}$, there exists a unique value of $m_{0}$ satisfying (4.8).

How does the presence of convex extraction costs directly affect the timing of mineral resource use? Does it affect the role played by the efficiency of the recycling technology of minerals embedded in green capital on the timing of investment in green capital?

To answer the former question, consider the present value of the marginal extraction cost of $m_{0}$. Notice first that when $\gamma>0$ and in the absence of discounting $\rho=0$, this cost is minimized by smoothing completely resource extraction $m_{0}=m_{1}=\frac{1}{2} M$. Under discounting, instead, this

\footnotetext{
${ }^{31}$ In practice we assume $\alpha=0$ to simplify the analysis and the notations. Numerical solutions suggest that the qualitative results extend to the case of an optimized use of finite fossil resources: this has been verified for the benchmark calibration (see footnote 22), with $c=1, \gamma=2$, and $\varepsilon$ varying from 0.1 to 3.1 .

${ }^{32}$ In the case of extraction costs for minerals, it is not necessarily the case that the stock $M$ is optimally exhausted. This is the case only for costs sufficiently low. Extraction costs are in turn partially endogenous. Here we focus the analysis on the first order condition.
} 
intertemporal smoothing is partial and $C\left(m_{0}\right)$ is minimized by partially shifting resource use to the future, i.e. $m_{0}=m_{0}^{c}<m_{1}=M-m_{0}^{c}$ since $\rho>0$. These two features provide the rationale for smoothing over time and partially delaying resource extraction when the marginal extraction cost is an increasing function of the extraction rate. Notice that these considerations intervene in our problem on the right-hand-side of (4.8), and are not directly affected by the possibility to recycle minerals, on which hinges the original mechanism put forward in this article. This remark provides the answer to the second question above: a marginal improvement in $\delta$ increases the present value of the marginal utility generated by first period mineral extraction and leads to an increase in optimal $m_{0}$, and investment in green capital, for any given schedule $C\left(m_{0}\right){ }^{33}$

We have shown that, although convex extraction costs introduce an economic incentive to smooth and actually postpone extraction of minerals, and therefore the build-up of the green capital stock, our original mechanism due to the possibility of recycling minerals embedded in green capital, is still at work, since it introduces a specific incentive to bring forward ceteris paribus minerals extraction and investment in green capital.

\subsection{Environmental damages from using fossil resources}

One of the main reasons underpinning the development of renewable energy production capacity around the world is the general recognition of the social costs resulting of the energy production from fossil resources. Since Smith (1972), the literature analyzing the interplay between recycling and pollution has focused on the potential limitation of local pollution from solid waste. Our original framework allows us to introduce an indirect link between the development of recycling and the reduction of greenhouse gas emissions, to mitigate climate change, a global pollution problem. ${ }^{34}$ Recycling influences the time profile of the energy mix. In the case of climate change related damages, the cumulative process of pollution raises the social payoff of early action. To take into account this additional factor affecting the optimal timing of investment in green capital, we extend our two-period model by assuming that using fossil resources also reduces utility. Specifically, we modify the utility function by adding a separable disutility term, convex in the current flow of pollution from the use of fossil resources: $d_{t} \frac{1}{\theta} f_{t}^{\theta}$, with $\theta>1$ and $t \in\{0 ; 1\}$. In order to capture two features of the climate change problem, we consider the case where it is not socially desirable to exhaust fossil resources, i.e. $f_{0}+f_{1}<F$, then consider that damages from first period emissions are relatively large, i.e. $d_{0} / d_{1}>1$ and study the impact of an increase in $d_{0} \cdot{ }^{35}$

\footnotetext{
${ }^{33}$ Formally, one can compute from (4.8) that $\frac{d m_{0}}{d \delta}=-\left(\frac{\partial P\left(m_{0}\right)}{\partial \delta}-\frac{\partial C\left(m_{0}\right)}{\partial \delta}\right) /\left(\frac{\partial P\left(m_{0}\right)}{\partial m_{0}}-\frac{\partial C\left(m_{0}\right)}{\partial m_{0}}\right)>0$, since, according to the previous analysis, the denominator is negative while $\frac{\partial C\left(m_{0}\right)}{\partial \delta}=0$ and $\frac{\partial P\left(m_{0}\right)}{\partial \delta}=$ $\frac{1}{1+\rho}\left(M-(1-\delta) m_{0}\right)^{-\varepsilon}\left(1+\varepsilon \frac{(1-\delta) m_{0}}{M-(1-\delta) m_{0}}\right)>0$.

${ }^{34}$ Though the use of fossil resources is a major cause of local pollution problems too, the crucial constraint on the supply of minerals for green capital (2.7) is potentially relevant on a global scale.

${ }^{35}$ Alternative setups to study the problem could be considered. One may impose a constraining ceiling $\bar{F}<F$, such that $f_{0}+f_{1} \leq \bar{F}$, in the spirit of the literature on "carbon budgets" (Chakravorty et al., 2006). Moreover, the cumulative nature of the pollution problem can be explicitly considered, by assuming that the second period disutility from pollution depends on past and present use of fossil resources. Numerical solutions of the case with cumulative pollution suggest that the qualitative results hold: this has been verified for the benchmark calibration (see footnote 22 ), with $d=1, \theta=2$, and $\varepsilon$ varying from 0.1 to 3.1 .
} 
The planner's program is modified, and writes

$$
\begin{aligned}
\max \frac{1}{1-\varepsilon}\left(f_{0}^{\alpha} m_{0}^{1-\alpha}\right)^{1-\varepsilon}-d_{0} \frac{1}{\theta} f_{0}^{\theta}+\frac{1}{1+\rho}\left(\frac{1}{1-\varepsilon}\left(f_{1}^{\alpha}\left(m_{1}+\delta m_{0}\right)^{1-\alpha}\right)^{1-\alpha}\right. & \left.-d_{1} \frac{1}{\theta} f_{1}^{\theta}\right) \\
& +\nu\left(M-m_{0}-m_{1}\right)
\end{aligned}
$$

We show in Appendix $\mathrm{F}$ that the interior solution (i.e. $m_{1}>0$ ) holds if $\delta<\widetilde{\delta} \equiv \frac{\widetilde{r}}{1+\widetilde{r}}$ and is defined by (4.2), (4.3) but for the following

$$
f_{0}=\left[\frac{\alpha}{d_{0}}\left(\frac{\frac{1}{1-\delta}}{1+\widetilde{r}} M\right)^{(1-\alpha)(1-\varepsilon)}\right]^{\frac{1}{\theta-\alpha(1-\varepsilon)}}, f_{1}=\left[\frac{\alpha}{d_{1}}\left(\frac{\widetilde{r}}{1+\widetilde{r}} M\right)^{(1-\alpha)(1-\varepsilon)}\right]^{\frac{1}{\theta-\alpha(1-\varepsilon)}}
$$

and the decline in energy consumption that is now given by

$$
\frac{q_{1}}{q_{0}}=\left[\left(\frac{d_{0}}{d_{1}}\right)^{\alpha}\left(\frac{1-\delta}{1+\rho}\right)^{\theta(1-\alpha)}\right]^{\frac{1}{\theta-(1-\varepsilon)[\alpha+\theta(1-\alpha)]}} .
$$

Since $\alpha \in(0,1), \varepsilon>0, \theta>1$ imply $\theta-(1-\varepsilon)[\alpha+\theta(1-\alpha)]>0$, the energy consumption declines at a faster pace with the efficiency of recycling $\left(\partial \frac{q_{1}}{q_{0}} / \partial \delta<0\right)$, and a slower rate with the importance of damages from initial polluting emissions $\left(\partial \frac{q_{1}}{q_{0}} / \partial d_{0}>0\right)$. As a consequence the gross discount rate $\tilde{r}$ falls with $\delta$ and increases with $d_{0}$ if $\varepsilon<1$, and vice versa. We find that our original mechanism is also operative in this framework. Similarly to the result in Proposition 2, the initial extraction rate of minerals and green investment increase with the recyclability, as well as the share of renewables in the energy mix in the first period (see Appendix F). In this case the energy mix is affected by $\delta$ also in the second period. In fact, improved recyclability of minerals embedded in green capital exerts the same effects discussed in detail in Section 4.1 in terms of the intertemporal allocation of fossil and mineral resources. However, in the present case the countervailing force due to the limited supply of fossil resources and their exhaustion is not active, since the total quantity of fossil resources used can vary. From (4.3) and (4.9) one can see that the use of fossil resources in the initial period moves with the recyclability in the same direction as renewables if the elasticity of the intertemporal substitution in consumption is larger than unity (i.e. $\varepsilon<1$ ), and vice versa, while fossil resource use in the second period always falls with $\delta$. Hence, improved recyclability of minerals allows society to reduce the total amount of fossil resources used when $\varepsilon>1$, and thus increase the share of renewables in the energy mix in both periods. ${ }^{36}$

How is investment in green capital affected by an increase in the damage of fossil resources use in the first period, i.e. $d_{0}$ ? This may represent a worsening of the climate change problem, as a short-cut for the cumulative nature of damages in such a pollution control problem. We find that an increase in $d_{0}$ does not necessarily put forward investment in green capital. In fact, for larger damage from fossils in the first period, the intuitive effect is that less fossil is used in the first period. As a consequence, the marginal utility of consumption in the first period is increased,

\footnotetext{
${ }^{36}$ This result would not hold if one were to adopt a "carbon budget" approach.
} 
making $m_{0}$ more valuable. This first effect calls for increasing $m_{0}$. However, a second effect, related to complementarity in production, comes from the fact that the marginal productivity of one resource increases with the use of the other resource. This calls for shifting the use of minerals to the second period, in order to postpone the use of the fossil resource. The balance between these two effects is solved according to the willingness of the representative agent to shift utility across time: if the elasticity of intertemporal substitution is sufficiently low, i.e. $\varepsilon>1$, the first effect dominates and $m_{0}$ increases with $d_{0}$, and vice versa.

\section{Conclusion}

Some observers argue that renewable energy is not manna from heaven, since it requires specific equipment that relies on intensive use of exhaustible and finite mineral resources. We have shown that this empirical fact favors abundant and early investment in green capital for the production of renewable energy, given that minerals embedded in specialized green capital can be recycled, as opposed to fossil resources burned for energy production.

Our analysis has focused on the role of recycling in determining the optimal path of extraction of fossil and mineral resources, and the investment in green capital. However, we have considered a constant, costless and exogenous recycling process. It would be relevant to check how robust our argument is to relaxing these assumptions. On its own the issue of the optimal choice of the recycling rate is interesting, and more so in our context as it could affect the timing of investment in green capital.

We have adopted the normative approach of the benevolent social planner. However, it can be argued that market failures would lead to inefficient equilibria. Some market failures concern imperfect competition, both in the primary resource market and in the secondary one, when there is recycling (see Ba and Mahenc, 2019, and the literature review therein). Other potential failures concern the thinness of markets for specific minerals and the joint production of several mineral resources (Fizaine, 2013). Moreover, the decentralized investment in R\&D directed at improvements in resource use efficiency or in recycling technology, may underpin potential dynamic inefficiencies (e.g. Zhou et al., 2018). Such market failures call for public intervention, raising the issue of their efficient design. We plan to study these extensions in future work.

It could also be interesting to investigate the role of the non-recycled share of used green capital. This cumulative waste involves a social cost to the extent that it may occupy scarce space or generate pollution in the absence of specific costly treatment. The social benefit of the development of recycling would therefore be confirmed : in addition to extending the life-cycle of the natural resource, therefore its use and the ability to generate energy from renewable sources, recycling reduces the amount of waste and its associated social cost. 


\section{References}

Acemoglu, D., Aghion, P., Bursztyn, L., and Hemous, D. (2012). The environment and directed technical change. American Economic Review, 102(1):131-66.

Ali, S. H., Giurco, D., Arndt, N., Nickless, E., Brown, G., Demetriades, A., Durrheim, R., Enriquez, M. A., Kinnaird, J., Littleboy, A., Meinert, L. D., Oberhänsli, R., Salem, J., Schodde, R., Schneider, G., Vidal, O., and Yakovleva, N. (2017). Mineral supply for sustainable development requires resource governance. Nature, 543(7645):367-372.

Ambec, S. and Crampes, C. (2019). Decarbonizing electricity generation with intermittent sources of energy. Journal of the Association of Environmental and Resource Economists, forthcoming.

Amigues, J.-P., Ayong Le Kama, A., and Moreaux, M. (2015). Equilibrium transitions from nonrenewable energy to renewable energy under capacity constraints. Journal of Economic Dynamics and Control, 55:89-112.

Amigues, J.-P., Favard, P., Gaudet, G., and Moreaux, M. (1998). On the optimal order of natural resource use when the capacity of the inexhaustible substitute is limited. Journal of Economic Theory, 80(1):153-170.

Arrobas, D. L. P., Ningthoujam, J., Mccormick, M. S., Drexhage, J. R., and Hund, K. L. (2017). The growing role of minerals and metals for a low carbon future. Technical Report 117581, The World Bank.

Ba, B. S. and Mahenc, P. (2019). Is recycling a threat or an opportunity for the extractor of an exhaustible resource? Environmental and Resource Economics, 73(4):1109-1134.

Boucekkine, R. and El Ouardighi, F. (2016). Optimal growth with polluting waste and recycling. In Dawid, H., Doerner, K. F., Feichtinger, G., Kort, P. M., and Seidl, A., editors, Dynamic Perspectives on Managerial Decision Making: Essays in Honor of Richard F. Hartl, Dynamic Modeling and Econometrics in Economics and Finance, pages 109-126. Springer International Publishing, Cham.

Chakravorty, U. and Krulce, D. L. (1994). Heterogeneous demand and order of resource extraction. Econometrica, 62(6):1445-1452.

Chakravorty, U., Magné, B., and Moreaux, M. (2006). A Hotelling model with a ceiling on the stock of pollution. Journal of Economic Dynamics and Control, 30(12):2875-2904.

Dasgupta, P., Gilbert, R. J., and Stiglitz, J. E. (1982). Invention and innovation under alternative market structures: The case of natural resources. The Review of Economic Studies, 49(4):567-582.

Dasgupta, P. S. and Heal, G. M. (1979). Economic Theory and Exhaustible Resources. Cambridge University Press.

Davison, R. (1978). Optimal Depletion of an Exhaustible Resource with Research and Development towards an Alternative Technology. The Review of Economic Studies, 45(2):355-367.

De Beir, J., Fodha, M., and Magris, F. (2010). Life cycle of products and cycles. Macroeconomic Dynamics, 14(2):212--230.

Di Vita, G. (2001). Technological change, growth and waste recycling. Energy Economics, 23(5):549567. 
Di Vita, G. (2007). Exhaustible resources and secondary materials: A macroeconomic analysis. Ecological Economics, 63(1):138-148.

DOE (2013). Ames laboratory to lead new research effort to address shortages in rare earth and other critical materials. Department of Energy, Public announcement of the establishment of the Critical Minerals Institute, January 9.

European Commission (2017). Study on the review of the list of critical raw materials. Technical report, Publications Office of the European Union.

European Commission (2018). Report on critical raw materials and the circular economy. Technical report, Commission Staff Working Document, SWD(2018) 36 final.

Fizaine, F. (2013). Byproduct production of minor metals: Threat or opportunity for the development of clean technologies? the pv sector as an illustration. Resources Policy, 38(3):373 383.

Fodha, M. and Magris, F. (2015). Recycling waste and endogenous fluctuations in an olg model. International Journal of Economic Theory, 11(4):405-427.

Goulder, L. H. and Schneider, S. H. (1999). Induced technological change and the attractiveness of co2 abatement policies. Resource and Energy Economics, 21(3):211 - 253.

Grimaud, A. and Rouge, L. (2008). Environment, directed technical change and economic policy. Environmental and Resource Economics, 41(4):439-463.

Hart, R. (2019). To everything there is a season: Carbon pricing, research subsidies, and the transition to fossil-free energy. Journal of the Association of Environmental and Resource Economists, $6(2): 135-175$.

Heal, G. M. (1993). Chapter 18 - The Optimal Use of Exhaustible Resources. In Kneese, A. V. and Sweeney, J. L., editors, Handbook of Natural Resource and Energy Economics, volume 3, pages 855-880. Elsevier.

Herfindahl, O. C. (1967). Depletion and economic theory. In Gaffney, M., editor, Extractive Resources and Taxation, pages 63-90. University of Wisconsin Press, Madison, WI.

Hertwich, E. G., Gibon, T., Bouman, E. A., Arvesen, A., Suh, S., Heath, G. A., Bergesen, J. D., Ramirez, A., Vega, M. I., and Shi, L. (2015). Integrated life-cycle assessment of electricity-supply scenarios confirms global environmental benefit of low-carbon technologies. Proceedings of the National Academy of Sciences, 112(20):6277-6282.

Hoel, M. (1978). Resource extraction and recycling with environmental costs. Journal of Environmental Economics and Management, 5(3):220-235.

Hotelling, H. (1931). The economics of exhaustible resources. Journal of Political Economy, $39(2): 137-175$.

Kamien, M. I. and Schwartz, N. L. (1978). Optimal exhaustible resource depletion with endogenous technical change. Review of Economic Studies, 45(1):179 - 196.

Kemp, M. C. and Van Long, N. (1980). On two folk theorems concerning the extraction of exhaustible resources. Econometrica, 48(3):663-673. 
Kverndokk, S. and Rosendahl, K. E. (2007). Climate policies and learning by doing: Impacts and timing of technology subsidies. Resource and Energy Economics, 29(1):58 - 82.

Lafforgue, G. and Rouge, L. (2019). A dynamic model of recycling with endogenous technological breakthrough. Resource and Energy Economics, 57:101-118.

Lemoine, D. and Traeger, C. (2014). Watch your step: Optimal policy in a tipping climate. American Economic Journal: Economic Policy, 6(1):137-66.

Lewis, T. R. (1982). Sufficient conditions for extracting least cost resource first. Econometrica, 50(4):1081-1083.

Moreaux, M. and Ricci, F. (2005). The simple analytics of developing resources from resources. Resource and Energy Economics, 27(1):41-63.

Moss, R. L., Tzimas, E., Kara, H., Willis, P., and Kooroshy, J. (2013). The potential risks from metals bottlenecks to the deployment of Strategic Energy Technologies. Energy Policy, 55(Supplement C):556-564.

Murto, P. and Nese, G. (2002). Input price risk and optimal timing of energy investment: choice between fossil- and biofuels. Working Papers in Economics 15/02, University of Bergen, Department of Economics.

Musu, I. and Lines, M. (1995). Endogenous growth and environmental preservation. In Boero, G. and Silberston, A., editors, Environmental Economics, Confederation of European Economic Associations, pages 273-295. Springer International Publishing.

Oren, S. S. and Powell, S. G. (1985). Optimal supply of a depletable resource with a backstop technology: Heal's theorem revisited. Operations Research, 33(2):277-292.

Pittel, K., Amigues, J.-P., and Kuhn, T. (2010). Recycling under a material balance constraint. Resource and Energy Economics, 32(3):379-394.

Pittel, K. and Bretschger, L. (2010). The implications of heterogeneous resource intensities on technical change and growth. Canadian Journal of Economics/Revue canadienne d'économique, 43(4):1173-1197.

Schulze, W. D. (1974). The optimal use of non-renewable resources: The theory of extraction. Journal of Environmental Economics and Management, 1:53-73.

Singer, D. A. (2017). Future copper resources. Ore Geology Reviews, 86(Supplement C):271-279.

Smith, V. L. (1972). Dynamics of waste accumulation: Disposal versus recycling. Quarterly Journal of Economics, 86(4):600-616.

Smulders, S. and de Nooij, M. (2003). The impact of energy conservation on technology and economic growth. Resource and Energy Economics, 25(1):59 - 79.

Tahvonen, O. and Salo, S. (2001). Economic growth and transitions between renewable and nonrenewable energy resources. European Economic Review, 45(8):1379-1398.

Tsur, Y. and Zemel, A. (2005). Scarcity, growth and r\&d. Journal of Environmental Economics and Management, 49(3):484-499. 
Vidal, O., Goffé, B., and Arndt, N. (2013). Metals for a low-carbon society. Nature Geoscience, 6(11):894-896.

Vidal, O., Rostom, F., François, C., and Giraud, G. (2017). Global trends in metal consumption and supply: The raw material-energy nexus. Elements, 13(5):319-324.

Vogt-Schilb, A., Meunier, G., and Hallegatte, S. (2018). When starting with the most expensive option makes sense: Optimal timing, cost and sectoral allocation of abatement investment. Journal of Environmental Economics and Management, 88:210 - 233.

Weinstein, M. and Zeckhauser, R. (1973). Critical ratios and efficient allocation. Journal of Public Economics, 2(2):147-157.

Wickart, M. and Madlener, R. (2007). Optimal technology choice and investment timing: A stochastic model of industrial cogeneration vs. heat-only production. Energy Economics, 29(4):934-952.

Wirl, F. (1988). Resource extraction of imperfect substitutes. Energy Economics, 10(3):242-248.

Zhou, S. L., Smulders, S., and Gerlagh, R. (2018). Closing the loop in a circular economy: Saving resources or suffocating innovations? Presented at SURED conference (Ascona, Switzerland). 


\section{A Solutions of the infinite horizon}

In the general case where we do not assume that there is an interval from $\underline{t}$ to $\bar{t}$ which corresponds to positive mineral extraction, the maximization program writes:

$$
\begin{aligned}
& \max _{f_{x}, m_{x}} \sum_{x \geq 0} \frac{(1+\rho)^{-x}}{1-\varepsilon}\left(f_{x}^{\alpha}\left(K_{-1} \delta^{x+1}+\sum_{u=0}^{x} m_{u} \delta^{x-u}\right)^{1-\alpha}\right)^{1-\varepsilon} \\
& +\lambda\left(F-\sum_{x \geq 0} f_{x}\right)+\nu\left(M-\sum_{x \geq 0} m_{x}\right)+\sum_{x \geq 0} \lambda_{x} f_{x}+\sum_{x \geq 0} \nu_{x} m_{x}
\end{aligned}
$$

In the following, we simplify the notations by introducing: $\phi:=\alpha(1-\varepsilon)$ and $\mu:=(1-\alpha)(1-\varepsilon)$. To solve the program, we first assume in subsection A.1 that the positivity constraints always hold after a certain date $\underline{t}$, i.e. $\forall t \geq \underline{t}, \lambda_{t}=\nu_{t}=0$, which corresponds to an endless extraction of resources. Then in subsection A.2, we derive the optimal solution in the case where minerals are depleted at the initial period: $\forall t>0, m_{t}=0$. We show in Appendix B that these solutions are indeed optimal under the conditions given in Proposition 1.

\section{A.1 Endless extraction}

We assume a positive extraction of both resources starting at a date $\underline{t}$, before which only fossils are extracted. Using (2.6), the social planner's program rewrites:

$$
\max \sum_{x=0}^{\underline{t}-1} \frac{(1+\rho)^{-x}}{1-\varepsilon} f_{x}^{\phi}\left(\delta^{x+1} K_{-1}\right)^{\mu}+\sum_{x \geq \underline{t}} \frac{(1+\rho)^{-x}}{1-\varepsilon} f_{x}^{\phi} K_{x}^{\mu}+\lambda\left(F-\sum_{x \geq 0} f_{x}\right)+\nu\left(M-\sum_{x \geq \underline{t}} m_{x}\right)
$$

In the computations, we assume $\delta>0$, but the solution extends to the limit cases $\delta=0$. The $\log$ case $\varepsilon=1$ is covered by the computations (only the program writes differently in this case).

The f.o.c.s are:

$$
\begin{cases}\left(\partial f_{t}\right)_{t<\underline{t}} & \alpha f_{t}^{\phi-1}\left(\delta K_{-1}\right)^{\mu}=\lambda\left(\frac{1+\rho}{\delta^{\mu}}\right)^{t} \\ \left(\partial f_{t}\right)_{t \geq \underline{t}} & \alpha f_{t}^{\phi-1} K_{t}^{\mu}=\lambda(1+\rho)^{t} \\ \left(\partial m_{t}\right)_{t \geq \underline{t}} & \sum_{x \geq t} f_{x}^{\phi} K_{x}^{\mu-1}\left(\frac{\delta}{1+\rho}\right)^{x}=\frac{\nu}{1-\alpha} \delta^{t}\end{cases}
$$

where the last f.o.c. uses the definition of $K_{x}$ in (2.6).

Subtracting the f.o.c. on $m_{t+1}$ from the f.o.c. on $m_{t}$, we have

$$
f_{t}^{\phi} K_{t}^{\mu-1}=\frac{\nu}{1-\alpha}(1-\delta)(1+\rho)^{t}
$$

so that,

$$
\forall t \geq \underline{t}, K_{t}=\left(\frac{\nu}{1-\alpha}(1-\delta)(1+\rho)^{t}\right)^{\frac{1}{\mu-1}} f_{t}^{\frac{\phi}{1-\mu}}
$$

Injecting this into the f.o.c. on $f_{t}$, and given that $\phi+\mu-1=-\varepsilon<0$ :

$$
\left\{\begin{array}{l}
\lambda=\alpha f_{0}^{\phi-1}\left(\delta K_{-1}\right)^{\mu} \\
\forall t<\underline{t}, f_{t}=\left(\frac{\lambda}{\alpha}\left(\frac{1+\rho}{\delta^{\mu}}\right)^{t}\left(\delta K_{-1}\right)^{-\mu}\right)^{\frac{1}{\phi-1}}=f_{0}\left(\frac{1+\rho}{\delta^{\mu}}\right)^{\frac{t}{\phi-1}} \\
\forall t \geq \underline{t}, f_{t}=\left(\frac{\lambda}{\alpha}(1+\rho)^{\frac{t}{1-\mu}}\left(\frac{\nu}{1-\alpha}(1-\delta)\right)^{\frac{\mu}{1-\mu}}\right)^{\frac{1-\mu}{\phi+\mu-1}}
\end{array}\right.
$$

Defining $r:=(1+\rho)^{\frac{1}{\phi+\mu-1}}=(1+\rho)^{-1 / \varepsilon}<1$ and $R:=\left(\frac{1+\rho}{\delta^{\mu}}\right)^{\frac{1}{\phi-1}}$, this system gives: 


$$
\forall t<\underline{t}, f_{t}=f_{0} R^{t} \text { and } \forall t \geq \underline{t}, f_{t}=f_{S} r^{t}
$$

Combining the f.o.c.s of $f_{\underline{t}}$ and $f_{\underline{t}-1}$, we have $f_{S}=f_{0}\left(r \delta \frac{K_{-1}}{K_{\underline{t}}}\right)^{\frac{\mu}{\phi-1}}$, so that

$$
\sum_{t \geq 0} f_{t}=f_{0} \frac{1-R^{\underline{t}}}{1-R}+f_{S} \frac{r^{\underline{t}}}{1-r}=f_{0}\left(\frac{1-R^{\underline{t}}}{1-R}+\left(r \delta \frac{K_{-1}}{K_{\underline{t}}}\right)^{\frac{\mu}{\phi-1}} \frac{r \underline{t}}{1-r}\right)=f_{S}\left(\left(r \delta \frac{K_{-1}}{K_{\underline{t}}}\right)^{\frac{\mu}{1-\phi}} \frac{1-R^{\underline{t}}}{1-R}+\frac{r \underline{\underline{t}}}{1-r}\right)
$$

The constraint (2.4) on recoverable resource of fossils gives $f_{0}=F\left(\frac{1-R^{\underline{\underline{t}}}}{1-R}+\left(r \delta \frac{K_{-1}}{K_{\underline{\underline{t}}}}\right)^{\frac{\mu}{\phi-1}} \frac{r^{\underline{\underline{t}}}}{1-r}\right)^{-1}$ and $f_{S}=$ $F\left(\left(r \delta \frac{K_{-1}}{K_{\underline{t}}}\right)^{\frac{\mu}{1-\phi}} \frac{1-R^{\underline{t}}}{1-R}+\frac{r_{\underline{\underline{t}}}}{1-r}\right)^{-1}$. Turning to the minerals, we have from (A.2) and (A.3), using (2.6):

$$
\begin{gathered}
m_{\underline{t}}=K_{\underline{t}}-\delta^{\underline{t}+1} K_{-1}=\left(\frac{\nu}{1-\alpha}(1-\delta)\right)^{\frac{1}{\mu-1}} f_{S}^{\frac{\phi}{1-\mu}} r^{\underline{t}}-\delta^{\underline{t}+1} K_{-1} \\
\forall t>\underline{t}, m_{t}=K_{t}-\delta K_{t-1}=\left(\frac{\nu}{1-\alpha}(1-\delta)\right)^{\frac{1}{\mu-1}} f_{S}^{\frac{\phi}{1-\mu}} r^{t-1}(r-\delta)=: K_{\underline{t}} r^{t-\underline{t}-1}(r-\delta)
\end{gathered}
$$

Lastly, $K_{\underline{t}}$ is determined by the transversality condition $(2.7)$ on $\left(m_{t}\right)_{t \geq 0}$ :

$$
M=\sum_{t \geq \underline{t}} m_{t}=K_{\underline{t}}-\delta^{\underline{t}+1} K_{-1}+\sum_{t>\underline{t}} K_{\underline{t}} r^{t-\underline{t}-1}(r-\delta)=K_{\underline{t}} \frac{1-\delta}{1-r}-\delta^{\underline{t}+1} K_{-1}
$$

i.e. $K_{\underline{t}}=\frac{1-r}{1-\delta}\left(M+\delta^{\underline{t}+1} K_{-1}\right)$. Finally, we obtain, with $r=(1+\rho)^{-\frac{1}{\varepsilon}}$ and $R=\left(\frac{1+\rho}{\delta^{\mu}}\right)^{\frac{1}{\phi-1}}$ :

$$
\begin{aligned}
\forall t<\underline{t}, \quad f_{t} & =\left(\frac{1-R^{\underline{t}}}{1-R}+\left(\frac{r \delta(1-\delta) K_{-1}}{(1-r)\left(M+\delta^{\underline{t}+1} K_{-1}\right)}\right)^{\frac{\mu}{\phi-1}} \frac{r^{\underline{t}}}{1-r}\right)^{-1} F \cdot R^{t} \\
\forall t \geq \underline{t}, \quad f_{t} & =\left(\left(\frac{r \delta(1-\delta) K_{-1}}{(1-r)\left(M+\delta^{\underline{t}+1} K_{-1}\right)}\right)^{\frac{\mu}{1-\phi}} \frac{1-R^{\underline{t}}}{1-R}+\frac{r^{\underline{t}}}{1-r}\right)^{-1} F \cdot r^{t} \\
\forall t<\underline{t}, \quad m_{t} & =0 \\
m_{\underline{t}} & =\frac{1-r}{1-\delta} M-\frac{r-\delta}{1-\delta} \delta^{\underline{t}+1} K_{-1} \\
\forall t>\underline{t}, \quad m_{t} & =\frac{1-r}{1-\delta}\left(M+\delta^{\underline{t}+1} K_{-1}\right)\left(1-\frac{\delta}{r}\right) r^{t-\underline{t}}
\end{aligned}
$$

The positivity constraints hold for $\delta<r$ and for $\underline{t}$ such that $M>\delta^{\underline{t}+1} K_{-1} \frac{r-\delta}{1-r}$.

\section{A.2 Immediate exhaustion}

In this case, $\forall t>0, m_{t}=0$. We also assume that $\forall t, f_{t}>0$ (see subsection B.1 for the justification). The objective is increasing in $m_{0}$, so it should be set to its maximum: $m_{0}=M$. Then, the f.o.c. on $f_{t}$ writes: $\alpha f_{t}^{\phi-1} M^{\mu} \delta^{\mu t}=\lambda(1+\rho)^{t}$, i.e. $f_{t}=\left(\frac{\lambda}{\alpha M^{\mu}}\left(\frac{1+\rho}{\delta^{\mu}}\right)^{t}\right)^{\frac{1}{\phi-1}}$. Defining $f_{0}:=\left(\frac{\lambda}{\alpha M^{\mu}}\right)^{\frac{1}{\phi-1}}$, we have: $\forall t \geq 0, \quad f_{t}=f_{0} R^{t}$. To conclude, notice that according to Lemma 1 in Appendix $\mathrm{C} \delta \geq r \Rightarrow R<1$. ${ }^{37}$ The transversality condition (2.4) must be saturated, as the program is increasing in $f_{t}$ for all $t$. This gives

Except in the degenerate case $\delta=1+\rho=R=1$ for which there is no solution because the supremum of the objective is infinite and cannot be attained. However $\rho>0$ by assumption. 
$F=\sum_{t \geq 0} f_{t}=\frac{f_{0}}{1-R}$, thus $f_{0}=F(1-R)$. Finally, we obtain: $\forall t \geq 0, \quad f_{t}=F(1-R) R^{t}$

\section{B Optimality of the solutions}

In this section, we demonstrate the results of Proposition 1. We show in B.1 that it is never optimal to interrupt the extraction when $\delta<r$. Then we derive in B.2 the solution when minerals are depleted in a finite time and show that it is sub-optimal. Finally, we use all this to prove Proposition 1 in the case $\delta<r$ in B.3, and we treat the case $\delta \geq r$ in B.4.

\section{B.1 Interruption of extraction}

It is never optimal to let $K$ or $f$ be nil at any period because the marginal welfare goes to $+\infty$ when consumption is nil. Let us now show that for $\delta<r$, it is never optimal to interrupt mineral extraction, i.e. $\delta<r \Longrightarrow \exists \underline{t}, \exists \bar{t} \geq \underline{t}, m_{t}>0 \Longleftrightarrow t \in[\underline{t}, \bar{t}]$. Let $\left(m_{t}, f_{t}\right)_{t \geq 0}$ be an optimal solution and let $T$ be such that $m_{T}>0$ and such that $\left\{t>T \mid m_{t}>0\right\} \neq \varnothing$. We define $\tau:=\min _{t>T}\left\{t \mid m_{t}>0\right\}$ in order to prove that $\tau=T+1$, i.e. that interruption of mineral extraction is suboptimal. Let us assume ad absurdo that $\tau \neq T+1$, so that $m_{T+1}=0$ and $m_{\tau-1}=0$. Then, as $\phi<1$ and $\varepsilon>0$, we deduce from $\delta<r$ using the definition of $r$ :

$$
\begin{aligned}
1>\left(\frac{\delta}{r}\right)^{(\tau-1-T) \frac{\varepsilon}{1-\phi}} & =(1+\rho)^{\frac{\tau-1-T}{1-\phi}}\left(\frac{K_{T}}{K_{T}} \cdot \delta^{\tau-1-T}\right)^{\frac{\varepsilon}{1-\phi}} \\
& =(1+\rho)^{\frac{\tau-1-T}{1-\phi}}\left(\frac{K_{\tau-1}}{K_{T}}\right)^{\frac{\mu \phi}{\phi-1}+1-\mu}=(1+\rho)^{(\tau-1-T)}\left(\frac{K_{T}}{K_{\tau-1}}\right)^{\mu-1}\left(\frac{f_{T}}{f_{\tau-1}}\right)^{\phi}
\end{aligned}
$$

where we used the f.o.c.s on $f_{T}$ and $f_{T+1}$ to find the last equality. We thus have

$$
(1-\alpha)(1+\rho)^{-T} f_{T}^{\phi} K_{T}^{\mu-1}<(1-\alpha)(1+\rho)^{-(\tau-1)} f_{\tau-1}^{\phi} K_{\tau-1}^{\mu-1} .
$$

Besides, taking into account the non-negativity constraints $\nu_{\tau-1} \geq 0$ and $\nu_{T+1} \geq 0$ in equation A.1, we have:

$$
(1-\alpha)(1+\rho)^{-T} f_{T}^{\phi} K_{T}^{\mu-1}=(1-\delta) \nu+\delta \nu_{T+1} \geq(1-\delta) \nu-\nu_{\tau-1}=(1-\alpha)(1+\rho)^{-(\tau-1)} f_{\tau-1}^{\phi} K_{\tau-1}^{\mu-1}
$$

The last two inequalities contradict, so we deduce that $\tau=T+1$.

\section{B.2 Exhaustion in a finite time}

Let $\bar{t}>0$ be the last period at which minerals are extracted. We assume in this subsection that extraction takes place from the initial period on. The program can be decomposed in two eras, during and after the extraction of minerals:

$$
\max \sum_{x=0}^{\bar{t}} \frac{(1+\rho)^{-x}}{1-\varepsilon} f_{x}^{\phi} K_{x}^{\mu}+\sum_{x>\bar{t}} \frac{(1+\rho)^{-x}}{1-\varepsilon} f_{x}^{\phi} K_{\bar{t}}^{\mu} \delta^{\mu(x-\bar{t})}+\lambda\left(F-\sum_{x \geq 0} f_{x}\right)+\nu\left(M-\sum_{x \geq 0} m_{x}\right)
$$

Using the f.o.c.s, one can derive the unique solution and write the inter-temporal welfare as follows, after defining with $a:=\frac{1-r^{\bar{t}}}{1-r}(1-\delta)$ and $c=\left((1-\delta) \frac{R^{\bar{t}}}{1-R}\right)^{\frac{\phi-1}{\mu+\phi-1}} \delta^{\frac{\mu \bar{t}}{\mu+\phi-1}}:^{38}$

$$
\begin{aligned}
W_{\bar{t}} & =\frac{f_{0}^{\phi} m_{0}^{\mu}}{1-\varepsilon}+\sum_{x=1}^{\bar{t}-1}(1+\rho)^{-x} \frac{f_{x}^{\phi} K_{x}^{\mu}}{1-\varepsilon}+(1+\rho)^{-\bar{t}} \frac{f_{\bar{t}}^{\phi} K_{\bar{t}}^{\mu}}{1-\varepsilon}+\sum_{x>\bar{t}}(1+\rho)^{-x} \frac{f_{x}^{\phi} K_{\bar{t}}^{\mu}}{1-\varepsilon} \delta^{\mu(x-\bar{t})} \\
& =\frac{1}{1-\varepsilon}\left(F^{\phi} \bar{M}^{\mu}(1-\delta)^{\phi-1}(a+c)^{1-\phi-\mu}\right)
\end{aligned}
$$

To show that extraction in a finite time is not optimal, we derive welfare with respect to the last period of

\footnotetext{
${ }^{38}$ The detailed derivation is available in the on-line Appendix. Referees please see Appendix G.
} 
extraction:

$$
\begin{aligned}
\frac{d W}{d \bar{t}} & =W \frac{1-\phi-\mu}{a+c} \frac{d(a+c)}{d \bar{t}} \\
\frac{a+c}{1-\phi-\mu} \frac{1}{W} \frac{d \ln W}{d \bar{t}} & =\frac{d a}{d \bar{t}}+\frac{d c}{d \bar{t}}=-\ln (r) r^{\bar{t}}\left(\frac{1-\delta}{1-r}-\left(\frac{1-\delta}{1-R}\right)^{\frac{\phi-1}{\mu+\phi-1}}\right)
\end{aligned}
$$

It is optimal to delay the exhaustion of minerals if and only if $\frac{d \ln W}{d t}>0$. Notice that

$$
\begin{gathered}
\frac{1}{W} \frac{d \ln W}{d \bar{t}}>0 \Longleftrightarrow \frac{1-\delta}{1-r}>\left(\frac{1-\delta}{1-R}\right)^{\frac{\phi-1}{\mu+\phi-1}} \Longleftrightarrow 1-R>(1-\delta)^{\frac{-\mu}{\phi-1}}(1-r)^{\frac{\mu+\phi-1}{\phi-1}} \\
\Longleftrightarrow 1-\frac{r^{\frac{\mu+\phi-1}{\phi-1}}}{\delta^{\frac{\mu}{\phi-1}}}>\left(\frac{1-\delta}{1-r}\right)^{\frac{\mu}{1-\phi}}(1-r)
\end{gathered}
$$

Defining $v:=\frac{\mu}{1-\phi}$ and $g_{r}(\delta):=1-\left(\frac{\delta}{r}\right)^{v} r-\left(\frac{1-\delta}{1-r}\right)^{v}(1-r)$, we have $\frac{1}{W} \frac{d \ln W}{d t}>0 \Longleftrightarrow g_{r}(\delta)>0$. Yet, $g_{r}^{\prime}(\delta)=v\left(\left(\frac{1-\delta}{1-r}\right)^{v-1}-\left(\frac{\delta}{r}\right)^{v-1}\right)$. For $v \in(0 ; 1): g_{r}^{\prime}(\delta)>0 \Longleftrightarrow \frac{1-\delta}{1-r}<\frac{\delta}{r} \Longleftrightarrow r<\delta$ while for $v<0$, the inverse is true: $g_{r}^{\prime}(\delta)>0 \Longleftrightarrow \delta<r$. In addition, $0<\varepsilon<1 \Longrightarrow(1-\varepsilon)-\alpha(1-\varepsilon)<1-\alpha(1-\varepsilon) \Longrightarrow$ $v=\frac{(1-\alpha)(1-\varepsilon)}{1-\alpha(1-\varepsilon)} \in(0 ; 1)$ while $\varepsilon>1 \Longrightarrow v<0$ (in the limit case $\left.\varepsilon=1, g_{r}=0\right)$. For $\varepsilon<1$, as $g_{r}(r)=0$ and $g_{r}$ is strictly decreasing below $r$ and strictly increasing above $r$, we deduce that $\forall r, g_{r} \geq 0$ and that $\forall r, \forall \delta \neq r, g_{r}(\delta)>0$. For $\varepsilon>1$, the same reasoning shows that $\forall r, g_{r} \leq 0$ and that $\forall r, \forall \delta \neq r, g_{r}(\delta)<0$. Given that $W>0 \Longleftrightarrow \varepsilon<1, \varepsilon \neq 1 \Longrightarrow \forall \delta \neq r, \frac{d \ln W}{d \bar{t}}>0$. The solutions extend to the $\log$ case $\varepsilon=1$, but the formula of intertemporal welfare does not. Let us compare in this case $W_{\bar{t}+1}$ and $W_{\bar{t}}$.

$$
\begin{aligned}
W_{\bar{t}+1}-W_{\bar{t}}= & \sum_{t=0}^{\bar{t}}(1+\rho)^{-t} \ln \left(\left(\bar{M} \frac{1-r}{1-\delta}\right)^{1-\alpha} F^{\alpha} r^{t}\right)+\sum_{t>\bar{t}}(1+\rho)^{-t} \ln \left(\left(\bar{M} \delta^{t-\bar{t}-1} r^{\bar{t}+1}\right)^{1-\alpha} F^{\alpha} r^{\alpha t}\right) \\
& -\sum_{t=0}^{\bar{t}-1}(1+\rho)^{-t} \ln \left(\left(\bar{M} \frac{1-r}{1-\delta}\right)^{1-\alpha} F^{\alpha} r^{t}\right)-\sum_{t \geq \bar{t}}(1+\rho)^{-t} \ln \left(\left(\bar{M} \delta^{t-\bar{t}} r^{\bar{t}}\right)^{1-\alpha} F^{\alpha} r^{\alpha t}\right) \\
\frac{W_{\bar{t}+1}-W_{\bar{t}}}{1-\alpha}= & r^{\bar{t}} \ln \left(\frac{1-\delta}{1-r}\right)+\sum_{t>\bar{t}} r^{t} \ln \left(\frac{\delta}{r}\right)=r^{\bar{t}}\left(\ln \left(\frac{1-r}{1-\delta}\right)+\frac{r}{1-r} \ln \left(\frac{r}{\delta}\right)\right)
\end{aligned}
$$

Hence, for $\varepsilon=1, W_{\bar{t}+1}>W_{\bar{t}} \Longleftrightarrow h_{r}(\delta):=(1-r) \ln \left(\frac{1-r}{1-\delta}\right)+r \ln \left(\frac{r}{\delta}\right)>0$. Yet, $h_{r}^{\prime}(\delta)=\frac{1-r}{1-\delta}-\frac{r}{\delta}>$ $0 \Longleftrightarrow \delta>r$ and $h_{r}(r)=0$, so that $\forall \delta \neq r, h_{r}(\delta)>0$. As a consequence, whatever the value of $\varepsilon$, it is always optimal to delay the end of mineral extraction and it is never optimal to exhaust minerals at a date $\bar{t}>0$. Indeed, in the only case for which it is optimal to do so, $\delta=r$, all candidate solutions conflate to immediate exhaustion.

\section{B.3 Case $\delta<r$}

In this subsection, we call $\underline{t}$ the first period for which an optimal program's mineral extraction has a positive value: $\underline{t}:=\min _{t \geq 0}\left\{m_{t}>\overline{0}\right\}$. Let us prove by induction on $\underline{t}$ that for all optimal solutions $\left(m_{t}, f_{t}\right)_{t \geq 0}$ such that $\underline{t}$ is the first period with positive mineral extraction, $t<\underline{t} \Longleftrightarrow m_{t}=0 . t<\underline{t} \Longrightarrow m_{t}=0$ being true by definition, we only need to prove the reciprocal. In the base case $\underline{t}=0, m_{t}=0 \Longrightarrow t \in \emptyset \Longrightarrow t<$ $\underline{t}=0$ comes from the results of the three previous subsections that it is never optimal to stop or interrupt extraction. Then we turn to the inductive step, and we assume that the proposition has been proven for all $\underline{t} \leq n$, to show it in the case $\underline{t}=n+1$. Let $\left(m_{t}, f_{t}\right)_{t>0}$ be a solution of the original program such that $\underline{t}=n+1$. Necessarily, $\left(m_{t}, f_{t}\right)_{t>1}$ is optimal solution of the program starting at 1 with stock of resources $\left(M-m_{0}, F-f_{0}\right)$. Applying the induction on $\left(m_{t}, f_{t}\right)_{t \geq 1}$, we know that $\forall t \geq 1,\left(m_{t}=0 \Longrightarrow t<n+1\right)$. In addition, by definition of $\underline{t}, m_{0}=0$, so that $\forall t \geq 0, t<\underline{t} \Longleftrightarrow m_{t}=0$, which achieves the proof. Given 
that it is never optimal to interrupt or stop mineral extraction, the optimal extraction path is the one derived in Appendix A.

\section{B.4 Case $\delta \geq r$}

For $\delta>r$, and using the f.o.c.s on $f_{t}$ and $f_{t+1}{ }^{39}$, we have:

$$
\begin{aligned}
\forall t, \quad 1<\left(\frac{\delta}{r}\right)^{\frac{\varepsilon}{1-\phi}} & \leq(1+\rho)^{\frac{1}{1-\phi}}\left(\frac{m_{t+1}}{K_{t}}+\delta\right)^{\frac{\varepsilon}{1-\phi}} \\
& =(1+\rho)^{\frac{-1}{\phi-1}}\left(\frac{K_{t+1}}{K_{t}}\right)^{\frac{\mu \phi}{\phi-1}+1-\mu}=(1+\rho)\left(\frac{K_{t}}{K_{t+1}}\right)^{\mu-1}\left(\frac{f_{t}}{f_{t+1}}\right)^{\phi} \\
\forall t, \quad(1+\rho)^{-t} f_{t}^{\phi} K_{t}^{\mu-1} & >(1+\rho)^{-(t+1)} f_{t+1}^{\phi} K_{t+1}^{\mu-1}
\end{aligned}
$$

Suppose ad absurdo that the optimal path $\left(m_{t}, f_{t}\right)_{t \geq 0}$ is such that there exists $T>0$ such that $m_{T}>0$.

Let $\left(\tilde{m}_{t}, \tilde{f}_{t}\right)_{t \geq 0}$ be an alternative path defined by $\forall t, \tilde{f}_{t}=f_{t}, \forall t \notin\{T ; 0\}, \tilde{m}_{t}=m_{t}, \tilde{m}_{0}=m_{0}+\eta$, $\tilde{m_{T}}=m_{T}-\eta$, for an arbitrary $\eta \in\left(0, m_{T}\right)$. Let us compare the welfares $W_{\eta}$ and $W$ given by $(\tilde{m}, \tilde{f})$ and $(m, f)$, respectively.

$$
\begin{aligned}
& \tilde{W}_{\eta}=\frac{1}{1-\varepsilon}\left(\sum_{t<T}(1+\rho)^{-t} f_{t}^{\phi}\left(K_{t}+\eta \delta^{t}\right)^{\mu}+\sum_{t \geq T}(1+\rho)^{-t} f_{t}^{\phi}\left(K_{t}+\eta\left(\delta^{t}-\delta^{t-T}\right)\right)^{\mu}\right) \\
& \underset{\eta \rightarrow 0^{+}}{=} \frac{1}{1-\varepsilon}\left(\sum_{t<T}(1+\rho)^{-t} f_{t}^{\phi} K_{t}^{\mu}\left(1+\mu \eta \frac{\delta^{t}}{K_{t}}\right)+\sum_{t \geq T}(1+\rho)^{-t} f_{t}^{\phi} K_{t}^{\mu}\left(1+\mu \eta \frac{\delta^{t}-\delta^{t-T}}{K_{t}}\right)\right)+o(\eta) \\
& \tilde{W}_{\eta}-W_{\eta} \underset{\eta \rightarrow 0^{+}}{=} \eta \alpha\left(\sum_{t \geq 0}\left((1+\rho)^{-t} f_{t}^{\phi} K_{t}^{\mu-1}-(1+\rho)^{-t-T} f_{t+T}^{\phi} K_{t+T}^{\mu-1}\right) \delta^{t}\right)+o(\eta)
\end{aligned}
$$

From above, we know that $\forall t,(1+\rho)^{-t} f_{t}^{\phi} K_{t}^{\mu-1}>(1+\rho)^{-t-T} f_{t+T}^{\phi} K_{t+T}^{\mu-1}$, which implies that $\tilde{W}_{\eta}>$ $W_{\eta}{ }^{40}$ This contradicts the optimality of $(m, f)$. We deduce that $\delta>r \Longrightarrow \forall T>0, m_{T}=0$. Observing that for $\delta=r$ the unconstrained solution gives $\forall t>0, m_{t}=0$ concludes the proof.

\section{Relations between the different rates}

Lemma 1. For $\varepsilon<1$, there are only three possible exclusive cases:

$$
\delta<R<r<\frac{1}{1+\rho} \quad \text { or } \quad r<R<\min \left\{\frac{1}{1+\rho}, \delta\right\} \quad \text { or } \quad \delta=R=r<\frac{1}{1+\rho}
$$

whereas for $\varepsilon>1$, the three possible cases are:

$$
\max \left\{\delta, \frac{1}{1+\rho}\right\}<r<R \quad \text { or } \quad \frac{1}{1+\rho}<R<r<\delta \quad \text { or } \quad \delta=R=r>\frac{1}{1+\rho}
$$

Finally, $\varepsilon=1$ entails $r=R=\frac{1}{1+\rho}<1$, with no relation on $\delta$.

As $\delta<1$, it follows that $\delta \geq r \Longrightarrow R<1$.

Proof. We always have $\delta<R \Longleftrightarrow \delta^{\phi-1}>\frac{r^{\mu+\phi-1}}{\delta^{\mu}} \Longleftrightarrow \delta<r$. For $\varepsilon<1, \mu=(1-\alpha)(1-\varepsilon)>0$, so

\footnotetext{
${ }^{39}$ To equate the f.o.c.s on $f_{t}$ we used the fact the $\forall t, f_{t}>0$ shown in subsection B.1.

${ }^{40}$ The argument does not rely on $\varepsilon \neq 1$, the limit case $\varepsilon=1$ has not been presented for simplicity.
} 
that $r<R \Longleftrightarrow r^{\phi-1}>\frac{r^{\mu+\phi-1}}{\delta^{\mu}} \Longleftrightarrow \delta>r$. Ad absurdo, we also have that $\delta<R \Longrightarrow R<r$ for $\varepsilon<1$. In effect, suppose that $\delta<R$ and $r \leq R$. From the inequalities above (which are also valid as non-strict inequalities), we deduce two contradictory properties: $\delta<r$ and $\delta \geq r$. Reciprocally, $r \leq R \Longrightarrow R \leq \delta$ for $\varepsilon<1$. In addition, as $(\delta=r \Longleftrightarrow r=R) \Longrightarrow(\delta=r \Longleftrightarrow R=\delta)$, there are only three possible exclusive cases in the case $\varepsilon<1: \delta<R<r \quad \vee \quad r<R<\delta \quad \vee \quad \delta=R=r$. Using a similar reasoning for $\varepsilon>1$, and given that in this case $r<R \Longleftrightarrow \delta<r$, we have $\varepsilon>1 \Longrightarrow \delta<r<R \quad \vee \quad R<r<\delta \quad \vee \quad \delta=R=r$. As $\delta<1$, it follows that $\delta \geq r \Longrightarrow R<1$. Finally, observing that $R<\frac{1}{1+\rho} \Longleftrightarrow \frac{1+\rho}{\delta^{\mu}}>(1+\rho)^{1-\phi} \Longleftrightarrow$ $(1+\rho)^{\phi}>\delta^{\mu} \Longleftrightarrow(1+\rho)^{\alpha(1-\varepsilon)}>\delta^{(1-\alpha)(1-\varepsilon)} \Longleftrightarrow \varepsilon<1$ and $r<\frac{1}{1+\rho} \Longleftrightarrow(1+\rho)^{1-\frac{1}{\varepsilon}}<1 \Longleftrightarrow \varepsilon<1$ concludes the proof.

\section{Proof of Proposition 2}

The problem is:

$$
\begin{aligned}
\max \frac{1}{1-\varepsilon}\left[\left(A_{0} f_{0}\right)^{\alpha}\left(B_{0} m_{0}\right)^{1-\alpha}\right]^{1-\varepsilon}+\frac{1}{1-\varepsilon} \frac{1}{1+\rho}\left[\left(A_{1} f_{1}\right)^{\alpha}\left(B_{1}\left(m_{1}+\delta m_{0}\right)\right)^{1-\alpha}\right]^{1-\varepsilon} & \\
& +\lambda\left(F-f_{0}-f_{1}\right)+\nu\left(M-m_{0}-m_{1}\right)
\end{aligned}
$$

The f.o.c.s are

$$
\begin{cases}\left(\partial f_{0}\right) & \alpha \frac{q_{0}^{1-\varepsilon}}{f_{0}}=\lambda \\ \left(\partial f_{1}\right) & \frac{\alpha}{1+\rho} \frac{q_{1}^{1-\varepsilon}}{f_{1}}=\lambda \\ \left(\partial m_{0}\right) & (1-\alpha) \frac{q_{0}^{1-\varepsilon}}{m_{0}}+\delta \frac{1-\alpha}{1+\rho} \frac{q_{1}^{1-\varepsilon}}{m_{1}+\delta m_{0}}=\nu \\ \left(\partial m_{1}\right) & \frac{1-\alpha}{1+\rho} \frac{q_{1}^{1-\varepsilon}}{m_{1}+\delta m_{0}}=\nu\end{cases}
$$

Combining the last two

$$
q_{0}^{1-\varepsilon} m_{1}=\left(\frac{1-\delta}{1+\rho} q_{1}^{1-\varepsilon}-\delta q_{0}^{1-\varepsilon}\right) m_{0}
$$

and using the exhaustion condition $m_{0}+m_{1} \leq M$ one gets $m_{0}$ and $m_{1}$ in (4.3) with the definition (4.2). Hence $m_{1}>0$, only for $\delta$ below the threshold $\widetilde{\delta} \equiv \frac{\widetilde{r}}{1+\widetilde{r}}$. Combining the first two f.o.c.s to get $f_{1}=\frac{1}{1+\rho} \frac{q_{1}^{1-\varepsilon}}{q_{0}^{1-\varepsilon}} f_{0}$, then using the fossil resource exhaustion constraint $f_{0}+f_{1} \leq F$, one gets $f_{0}$ and $f_{1}$ in (4.3). As the Lagrangian is concave, the uniqueness stems from the Karush-Kuhn-Tucker theorem. These pace of resource use imply a specific pace of growth of energy consumption, given by (4.4). The gross discount factor is therefore:

$$
\tilde{r}=\left[\left(\frac{A_{1}}{A_{0}}\right)^{\alpha}\left(\frac{B_{1}}{B_{0}}\right)^{1-\alpha}\right]^{\frac{1-\varepsilon}{\varepsilon}}(1+\rho)^{-\frac{1}{\varepsilon}}(1-\delta)^{(1-\alpha) \frac{1-\varepsilon}{\varepsilon}}
$$

Notice that it is not affected by technological progress in the case of logarithmic utility, i.e. if $\varepsilon=1$. The prospect of higher resource productivity $\left(A_{1}>A_{0}\right.$ or $\left.B_{1}>B_{0}\right)$ increases the gross discount factor if the elasticity of intertemporal substitution is high enough $(1 / \varepsilon>1)$, and vice versa. The input ratios are given by $\frac{f_{0}}{K_{0}}=(1-\delta) \frac{F}{M}$ and $\frac{f_{1}}{K_{1}}=\frac{F}{M}$, implying $\frac{\partial f_{0} / K_{0}}{\partial \delta}<0$ but $\frac{\partial f_{1} / K_{1}}{\partial \delta}=0$. Besides, $\frac{K_{1}}{K_{0}}=\frac{m_{1}+\delta m_{0}}{m_{0}}=\widetilde{r}(1-\delta)=(1-\delta)^{1+\frac{1-\alpha}{\varepsilon}(1-\varepsilon)}\left[\left(\frac{A_{1}}{A_{0}}\right)^{\alpha}\left(\frac{B_{1}}{B_{0}}\right)^{1-\alpha}\right]^{\frac{1-\varepsilon}{\varepsilon}}(1+\rho)^{-\frac{(1-\varepsilon)}{\varepsilon}-1}$, implying $\frac{\partial \frac{K_{1}}{\partial \delta}}{\partial \delta}<0$. Rewrite $m_{0}=\frac{1}{(1-\delta)(1+\widetilde{r})} M$, to compute $\frac{\partial m_{0}}{\partial \delta}=-\frac{\frac{\partial(1-\delta)}{\partial \delta}+\frac{\partial(1-\delta) \tilde{r}}{\partial \delta}}{(1-\delta)(1+\tilde{r})} m_{0}>0$, since $\frac{\partial(1-\delta) \tilde{r}}{\partial \delta}=-\frac{1-\alpha(1-\varepsilon)}{1-\delta} \tilde{r}<0$. We can see that expectations of technological progress in both energy transformation technologies, postpone resource extraction, thus investment in green capital if the elasticity of intertemporal substitution is larger than unity: $\frac{\partial m_{0}}{\partial B_{1}}=-\frac{\frac{\partial(1-\delta) \tilde{r}}{\partial \delta}}{(1-\delta)(1+\widetilde{r})} m_{0}<0 \Leftrightarrow \varepsilon<1$. 


\section{E Analysis of the CES case in Section 4.2}

The problem is:

$$
\begin{array}{r}
\max \frac{1}{1-\varepsilon}\left(\alpha f_{0}^{\frac{\sigma-1}{\sigma}}+(1-\alpha) m_{0}^{\frac{\sigma-1}{\sigma}}\right)^{\frac{\sigma}{\sigma-1}(1-\varepsilon)}+\frac{1}{1-\varepsilon} \frac{1}{1+\rho}\left(\alpha f_{1}^{\frac{\sigma-1}{\sigma}}+(1-\alpha)\left(\delta m_{0}+m_{1}\right)^{\frac{\sigma-1}{\sigma}}\right)^{\frac{\sigma}{\sigma-1}(1-\varepsilon)} \\
+\lambda\left(F-f_{0}-f_{1}\right)+\nu\left(M-m_{0}-m_{1}\right)
\end{array}
$$

with $m_{0}, m_{1}, f_{0}$, and $f_{1} \geq 0$, and where $\sigma>0$ (Cobb-Douglas production function for $\left.\sigma=1\right)$ and $\varepsilon>0$ (log utility for $\varepsilon=1$ ).

A candidate interior solution satisfies the f.o.c.s

$$
\begin{cases}\left(\partial f_{0}\right) & f_{0}^{\frac{\sigma-1}{\sigma}-1} q_{0}^{1-\varepsilon-\frac{\sigma-1}{\sigma}}=\frac{\lambda}{\alpha} \\ \left(\partial f_{1}\right) & \frac{1}{1+\rho} f_{1}^{\frac{\sigma-1}{\sigma}}-1 q_{1}^{1-\varepsilon-\frac{\sigma-1}{\sigma}}=\frac{\lambda}{\alpha} \\ \left(\partial m_{0}\right) & m_{0}^{\frac{\sigma-1}{\sigma}-1} q_{0}^{1-\varepsilon-\frac{\sigma-1}{\sigma}}+\frac{\delta}{1+\rho}\left(\delta m_{0}+m_{1}\right)^{\frac{\sigma-1}{\sigma}-1} q_{1}^{1-\varepsilon-\frac{\sigma-1}{\sigma}}=\frac{\nu}{1-\alpha} \\ \left(\partial m_{1}\right) & \frac{1}{1+\rho}\left(\delta m_{0}+m_{1}\right)^{\frac{\sigma-1}{\sigma}-1} q_{1}^{1-\varepsilon-\frac{\sigma-1}{\sigma}}=\frac{\nu}{1-\alpha}\end{cases}
$$

Combine the two f.o.c.s on $f$ and the resource constraint $f_{0}+f_{1} \leq F$ to get $f_{0}$ and $f_{1}$ in (4.3), given the definition (4.5). Combine the two f.o.c.s on $m$ and the resource constraint $m_{0}+m_{1} \leq M, m_{0}$ and $m_{1}$ in (4.6). An interior solution requires $m_{1}>0 \Leftrightarrow\left(\frac{q_{1}}{q_{0}}\right)^{1-\varepsilon \sigma}(1+\rho)^{-\sigma}(1-\delta)^{\sigma}>\delta$, which defines the threshold on $\delta$ specified in the main text.

In the case of an interior solution, the consumption growth ratio is computed from

$$
\frac{q_{1}}{q_{0}}=\left(\frac{\alpha f_{1}^{\frac{\sigma-1}{\sigma}}+(1-\alpha)\left(m_{1}+\delta m_{0}\right)^{\frac{\sigma-1}{\sigma}}}{\alpha f_{0}^{\frac{\sigma-1}{\sigma}}+(1-\alpha) m_{0}^{\frac{\sigma-1}{\sigma}}}\right)^{\frac{\sigma}{\sigma-1}}
$$

substituting $f_{0}$ and $f_{1}$ from (4.3) and $m_{0}, m_{1}$ from (4.6) to define the intertemporal consumption ratio as the solution of the implicit function (4.7).

\section{F Damages from fossil resources}

Assuming that the resource constraint on fossils is not binding, the program writes:

$$
\max \frac{\left(f_{0}^{\alpha} m_{0}^{1-\alpha}\right)^{1-\varepsilon}}{1-\varepsilon}-\frac{d_{0}}{\theta} f_{0}^{\theta}+\frac{1}{1+\rho}\left(\frac{\left(f_{1}^{\alpha}\left(m_{1}+\delta m_{0}\right)^{1-\alpha}\right)^{1-\varepsilon}}{1-\varepsilon}-\frac{d_{1}}{\theta} f_{1}^{\theta}\right)+\nu\left(M-m_{0}-m_{1}\right)
$$

Using the definition $q_{t} \equiv f_{t}^{\alpha} K_{t}^{1-\alpha}$, the f.o.c.s are

$$
\begin{cases}\left(\partial f_{0}\right) & \alpha q_{0}^{1-\varepsilon}=d_{0} f_{0}^{\theta} \\ \left(\partial f_{1}\right) & \alpha q_{1}^{1-\varepsilon}=d_{1} f_{1}^{\theta} \\ \left(\partial m_{0}\right) & (1-\alpha) \frac{q_{0}^{1-\varepsilon}}{m_{0}}+(1-\alpha) \frac{\delta}{1+\rho} \frac{q_{1}^{1-\varepsilon}}{m_{1}+\delta m_{0}}=\nu \\ \left(\partial m_{1}\right) & (1-\alpha) \frac{1}{1+\rho} \frac{q_{1}^{1-\varepsilon}}{m_{1}+\delta m_{0}}=\nu\end{cases}
$$

The two f.o.c.s on $m$ together with the exhaustion of minerals $m_{1}+m_{0}=M$, give the solutions in (4.3) for $m_{0}$ and $m_{1}$, with $\widetilde{r}$ defined in (4.2). It follows that $K_{1}=\frac{\widetilde{r}}{1+\widetilde{r}} M$ and that an interior solution holds only if $\widetilde{r}>\frac{\delta}{1-\delta}$, i.e. $\delta<\tilde{\delta} \equiv \frac{\widetilde{r}}{1+\widetilde{r}}$. The two f.o.c.s on $f$ with the values of $m_{0}$ and $m_{1}$ in $(4.3)$ give $f_{0}$ and $f_{1}$ in 
(4.9). Using these expressions one gets

$$
\frac{q_{1}}{q_{0}}=\left[\left(\frac{d_{0}}{d_{1}}\right)^{\alpha}((1-\delta) \widetilde{r})^{\theta(1-\alpha)}\right]^{\frac{1}{\theta-\alpha(1-\varepsilon)}}
$$

Substituting (4.2) for $\widetilde{r}$ leads to (4.10).

Concerning the impact of a marginal increase of $\delta$ in the case of an interior solution, proceed as follows. For $m_{0}$, first combine (4.2) and (4.10) to compute $(1-\delta) \widetilde{r}=\left[\left(\frac{d_{0}}{d_{1}}\right)^{(1-\varepsilon) \alpha}\left(\frac{1-\delta}{1+\rho}\right)^{\theta-(1-\varepsilon) \alpha}\right]^{\frac{1}{\theta-(1-\varepsilon)[\alpha+\theta(1-\alpha)]}}$. Since $\theta-(1-\varepsilon)[\alpha+\theta(1-\alpha)]>0$ and $\theta-(1-\varepsilon) \alpha>0, \frac{\partial(1-\delta) \widetilde{r}}{\partial \delta}<0$. Next from (4.3) write $m_{0}=$ $\frac{1}{(1-\delta)(1+\widetilde{r})} M$. We get $\frac{\partial m_{0}}{\partial \delta}=-\frac{\frac{\partial(1-\delta)}{\partial \delta}+\frac{\partial(1-\delta) \widetilde{r}}{\partial \delta}}{(1-\delta)(1+\widetilde{r})} m_{0}>0$. Given the exhaustion of minerals, the effect on $m_{1}$ runs in opposite direction. From (4.9), we have that $\frac{\partial f_{0}}{\partial \delta}=\frac{(1-\alpha)(1-\varepsilon)}{\theta-\alpha(1-\varepsilon)} \frac{f_{0}}{m_{0}} \frac{\partial m_{0}}{\partial \delta}>0 \Leftrightarrow \varepsilon<1$, hence $\partial \frac{f_{0}}{K_{0}} / \partial \delta=-\frac{1}{m_{0}^{2}}\left(f_{0} \frac{\partial m_{0}}{\partial \delta}-m_{0} \frac{\partial f_{0}}{\partial \delta}\right)=-\frac{f_{0}}{m_{0}^{2}}\left(\frac{\theta+\varepsilon-1}{\theta-\alpha(1-\varepsilon)}\right) \frac{\partial m_{0}}{\partial \delta}<0$. Furthermore $\frac{\partial f_{1}}{\partial \delta}=\frac{(1-\alpha)(1-\varepsilon)}{\theta-\alpha(1-\varepsilon)} \frac{f_{1}}{\widetilde{r}(1+\widetilde{r})} \frac{\partial \widetilde{r}}{\partial \delta}<$ 0 since $\frac{\partial \widetilde{r}}{\partial \delta}<0 \Leftrightarrow \varepsilon<1$, and $\frac{f_{1}}{f_{0}}=\left(\left(\frac{d_{0}}{d_{1}}\right)^{\frac{\theta-(1-\varepsilon) \theta(1-\alpha)}{\theta-(1-\varepsilon)[\alpha+\theta(1-\alpha)]}}\left(\frac{1-\delta}{1+\rho}\right)^{\frac{\theta-(1-\varepsilon) \alpha}{\theta-(1-\varepsilon)[\alpha+\theta(1-\alpha)]}}\right)^{\frac{(1-\alpha)(1-\varepsilon)}{\theta-\alpha(1-\varepsilon)}}$, thus $\partial \frac{f_{1}}{f_{0}} / \partial \delta>$ $0 \Leftrightarrow \varepsilon>1$. Finally $\partial \frac{f_{1}}{K_{1}} / \partial \delta=-\frac{\theta+\varepsilon-1}{\theta-\alpha(1-\varepsilon)} \frac{M}{\widetilde{r}} \frac{f_{1}}{K_{1}} \frac{\partial \widetilde{r}}{\partial \delta}>0 \Leftrightarrow \varepsilon<1$.

Concerning the impact of a marginal increase in first period damages, in the case of an interior solution, we have the following. From $(4.9), \frac{\partial f_{0}}{\partial d_{0}}=\frac{f_{0}}{\theta-\alpha(1-\varepsilon)}\left((1-\alpha)(1-\varepsilon) \frac{\partial m_{0} / \partial d_{0}}{m_{0}}-\frac{1}{d_{0}}\right)<0$. However from the expressions above, it follows that $\frac{\partial m_{0}}{\partial d_{0}}=-\frac{\partial(1-\delta) \widetilde{r} / \partial d_{0}}{(1-\delta)(1+\widetilde{r})} m_{0}<0 \Leftrightarrow \varepsilon<1$. Finally the signs of the derivatives are summarized in Table 1.

Table 1: Signs of derivatives of the solutions with respect to the parameters

\begin{tabular}{ccccccc}
\hline \hline & $m_{0}$ & $m_{1}$ & $f_{0}$ & $f_{1}$ & $\frac{f_{0}}{K_{0}}$ & $\frac{f_{1}}{K_{1}}$ \\
\hline$\delta$ & + & - & $-\Longleftrightarrow \varepsilon>1$ & - & - & $-\Longleftrightarrow \varepsilon>1$ \\
$d_{0}$ & $+\Longleftrightarrow \varepsilon>1$ & $-\Longleftrightarrow \varepsilon>1$ & $\varepsilon<1 \Longrightarrow-$ & + & $\varepsilon>1 \Longrightarrow-$ & $+\Longleftrightarrow \varepsilon>1$ \\
$d_{1}-$ & $\Longleftrightarrow \varepsilon>1$ & $+\Longleftrightarrow \varepsilon>1$ & + & $\varepsilon<1 \Longrightarrow-$ & $\Longleftrightarrow \Longleftrightarrow \varepsilon>1$ & $\varepsilon>1 \Longrightarrow-$ \\
\hline \hline
\end{tabular}

\section{G On-line Appendix: Derivation of $W_{\bar{t}}$ in Appendix B.2}

The problem is

$$
\max \sum_{x=0}^{\bar{t}} \frac{(1+\rho)^{-x}}{1-\varepsilon} f_{x}^{\phi} K_{x}^{\mu}+\sum_{x>\bar{t}} \frac{(1+\rho)^{-x}}{1-\varepsilon} f_{x}^{\phi} K_{\bar{t}}^{\mu} \delta^{\mu(x-\bar{t})}+\lambda\left(F-\sum_{x \geq 0} f_{x}\right)+\nu\left(M-\sum_{x \geq 0} m_{x}\right)
$$

and the f.o.c.s are:

$$
\begin{cases}\left(\partial f_{t}\right)_{t \leq \bar{t}} & \alpha f_{t}^{\phi-1} K_{t}^{\mu}(1+\rho)^{-t}=\lambda \\ \left(\partial f_{t}\right)_{t>\bar{t}} & \alpha f_{t}^{\phi-1} K_{\bar{t}}^{\mu} \delta^{\mu(t-\bar{t})}(1+\rho)^{-t}=\lambda \\ \left(\partial m_{t}\right)_{t \leq \bar{t}} & (1-\alpha) \sum_{x=t}^{\bar{t}}(1+\rho)^{-x} f_{x}^{\phi} K_{x}^{\mu-1} \delta^{x-t}+(1-\alpha) \sum_{x>\bar{t}}(1+\rho)^{-x} f_{x}^{\phi} K_{\bar{t}}^{\mu-1} \delta^{\mu(x-\bar{t})+\bar{t}-t}=\nu\end{cases}
$$


The f.o.c. on $m_{t+1}$ can be expressed as follows for $t<\bar{t}$ :

$$
\begin{aligned}
\frac{1-\alpha}{\delta} \sum_{x=t}^{\bar{t}}(1+\rho)^{-x} & f_{x}^{\phi} K_{x}^{\mu-1} \delta^{x-t}-(1-\alpha)(1+\rho)^{-t} \frac{f_{t}^{\phi} K_{t}^{\mu-1}}{\delta} \\
+ & \frac{1-\alpha}{\delta} \sum_{x>\bar{t}}(1+\rho)^{-x} f_{x}^{\phi} K_{\bar{t}}^{\mu-1} \delta^{\mu(x-\bar{t})+\bar{t}-t}=\nu
\end{aligned}
$$

Then, $\forall t<\bar{t}, \frac{\left(\partial m_{t}\right)}{\delta}-\left(\partial m_{t+1}\right)$ yields $(1-\alpha)(1+\rho)^{-t} f_{t}^{\phi} K_{t}^{\mu-1}=\nu(1-\delta)$, so that

$$
\forall t<\bar{t}, \quad K_{t}=\left(\frac{\nu}{1-\alpha}(1-\delta)(1+\rho)^{t}\right)^{\frac{1}{\mu-1}} f_{t}^{\frac{\phi}{1-\mu}}
$$

Furthermore, $\left(\partial m_{\bar{t}}\right)$ gives $(1-\alpha) \sum_{x \geq \bar{t}}(1+\rho)^{-x} f_{x}^{\phi} K_{\bar{t}}^{\mu-1} \delta^{\mu(x-\bar{t})}=\nu$, i.e.

$$
K_{\bar{t}}=\left(\frac{1-\alpha}{\nu} \sum_{x \geq \bar{t}}(1+\rho)^{-x} f_{x}^{\phi} \delta^{\mu(x-\bar{t})}\right)^{\frac{1}{1-\mu}}
$$

Injecting (G.1) into the f.o.c. on $f_{t}$ yields $\forall t<\bar{t}$,

$$
\begin{aligned}
\lambda & =\alpha f_{t}^{\phi-1+\frac{\phi \mu}{1-\mu}}\left(\frac{\nu}{1-\alpha}(1-\delta)\right)^{\frac{\mu}{\mu-1}}(1+\rho)^{\frac{1}{\mu-1} t} \\
& =\alpha f_{0}^{\phi-1+\frac{\phi \mu}{1-\mu}}\left(\frac{\nu}{1-\alpha}(1-\delta)\right)^{\frac{\mu}{\mu-1}}
\end{aligned}
$$

i.e.

$$
f_{t}=\left(\frac{\lambda}{\alpha}(1+\rho)^{\frac{1}{1-\mu} t}\left(\frac{\nu}{1-\alpha}(1-\delta)\right)^{\frac{\mu}{1-\mu}}\right)^{\frac{1-\mu}{\phi+\mu-1}}
$$

Defining $r:=(1+\rho)^{\frac{1}{\phi+\mu-1}}<1$ and $f_{<}:=\left(\frac{\lambda}{\alpha}\right)^{\frac{1-\mu}{\phi+\mu-1}}\left(\frac{\nu}{1-\alpha}(1-\delta)\right)^{\frac{\mu}{\phi+\mu-1}}$, we have:

$$
\forall t<\bar{t}, \quad f_{t}=f_{<} r^{t}
$$

For $t>\bar{t}$, the f.o.c. on $f_{t}$ yields: ${ }^{41}$

$$
f_{t}=\left(\frac{\lambda \delta^{\mu \bar{t}}}{\alpha K_{\bar{t}}^{\mu}}\left(\frac{1+\rho}{\delta^{\mu}}\right)^{t}\right)^{\frac{1}{\phi-1}}
$$

Defining $R:=\left(\frac{1+\rho}{\delta^{\mu}}\right)^{\frac{1}{\phi-1}}$ and $f_{>}:=\left(\frac{\lambda \delta^{\mu \bar{t}}}{\alpha K_{\bar{t}}^{\mu}}\right)^{\frac{1}{\phi-1}}$, we have:

$$
\forall t>\bar{t}, \quad f_{t}=f_{>} R^{t}
$$

In the following, we assume that $R<1$. Combining the f.o.c. on $f_{\bar{t}}$ with the f.o.c. on $f_{\bar{t}+1}: f_{\bar{t}}^{\phi-1}(1+\rho)=$ $f_{\bar{t}+1}^{\phi-1} \delta^{\mu}=f_{>}^{\phi-1} R^{(\phi-1)(\bar{t}+1)} \delta^{\mu}$. This gives $f_{\bar{t}}$ :

$$
f_{\bar{t}}=f_{>} R^{\bar{t}}
$$

The transversality condition on fossils gives:

\footnotetext{
${ }^{41}$ For $\delta=0$, marginal welfare goes to infinity for each $t>\bar{t}$, as $K_{t}=0$. Hence, it is obviously suboptimal not to extract at every period in this case. In the following, we assume $\delta>0$.
} 


$$
F=f_{<} \sum_{t=0}^{\bar{t}-1} r^{t}+f_{>} \sum_{t \geq \bar{t}} R^{t}=f_{<} \frac{1-r^{\bar{t}}}{1-r}+f_{>} \frac{R^{\bar{t}}}{1-R}
$$

Injecting (G.3) into (G.1) for $t<\bar{t}$, we obtain $K_{t}=\left(\frac{\nu}{1-\alpha}(1-\delta)(1+\rho)^{t}\right)^{\frac{1}{\mu-1}} f_{<}^{\frac{\phi}{1-\mu}} r^{\frac{\phi}{1-\mu} t}$ so that $K_{0}=$ $\left(\frac{\nu}{1-\alpha}(1-\delta)\right)^{\frac{1}{\mu-1}} f_{<}^{\frac{\phi}{1-\mu}}$. Hence:

$$
\forall t<\bar{t}, \quad K_{t}=K_{0}(1+\rho)^{\frac{t}{\mu-1}} r^{\frac{\phi}{1-\mu} t}=K_{0}(1+\rho)^{\frac{1}{\phi+\mu-1} t}=K_{0} r^{t}
$$

which gives

$$
\forall t \in[1, \bar{t}-1], \quad m_{t}=K_{t}-\delta K_{t-1}=K_{0}\left(1-\frac{\delta}{r}\right) r^{t}
$$

The transversality condition on minerals gives:

$$
\begin{aligned}
M & =\sum_{t=0}^{\bar{t}} m_{t}=K_{0}-\delta K_{-1}+\sum_{t=1}^{\bar{t}-1} K_{0} r^{t}\left(1-\frac{\delta}{r}\right)+m_{\bar{t}} \\
m_{\bar{t}} & =M+\delta K_{-1}-K_{0}\left(1+(r-\delta) \frac{1-r^{\bar{t}-1}}{1-r}\right)
\end{aligned}
$$

Hence,

$$
\begin{aligned}
K_{\bar{t}} & =m_{\bar{t}}+\delta K_{\bar{t}-1}=M+\delta K_{-1}-K_{0}\left(1+(r-\delta) \frac{1-r^{\bar{t}-1}}{1-r}\right)+\delta K_{0} r^{\bar{t}-1} \\
& =M+\delta K_{-1}-K_{0} \frac{1-r^{\bar{t}}}{1-r}(1-\delta)=\bar{M}-a K_{0}
\end{aligned}
$$

with $a:=\frac{1-r^{\bar{t}}}{1-r}(1-\delta)$ and $\bar{M}:=M+\delta K_{-1}$.

Using (G.3) in the f.o.c. on $f_{0}$ together with the f.o.c. on $f_{\bar{t}+1}$ :

$$
f_{<}^{\phi-1} K_{0}^{\mu}=\delta^{-\mu \bar{t}} f_{>}^{\phi-1} K_{\bar{t}}^{\mu}
$$

Injecting (G.6) and (G.8) into this: ${ }^{42}$

$$
\begin{aligned}
& f_{<}^{\frac{\phi-1}{\mu}} f_{>}^{-\frac{\phi-1}{\mu}} K_{0}=\delta^{-\bar{t}}\left(\bar{M}-a K_{0}\right) \\
& f_{<}^{\frac{\phi-1}{\mu}}\left(\left(F-f_{<} \frac{1-r^{\bar{t}}}{1-r}\right) \frac{1-R}{R^{\bar{t}}}\right)^{-\frac{\phi-1}{\mu}}=\delta^{-\bar{t}}\left(\frac{\bar{M}}{K_{0}}-a\right)
\end{aligned}
$$

The f.o.c. on $m_{\bar{t}}$ gives:

$$
\begin{aligned}
(1-\alpha)(1+\rho)^{-\bar{t}} f_{\bar{t}}^{\phi} K_{\bar{t}}^{\mu-1}+(1-\alpha) \sum_{x>\bar{t}}(1+\rho)^{-x} f_{x}^{\phi} K_{\bar{t}}^{\mu-1} \delta^{\mu(x-\bar{t})} & =(1-\alpha) \sum_{x \geq \bar{t}} R^{x} f_{>}^{\phi} K_{\bar{t}}^{\mu-1} \delta^{-\mu \bar{t}} \\
& =(1-\alpha) f_{>}^{\phi} K_{\bar{t}}^{\mu-1} \delta^{-\mu \bar{t}} \frac{R^{\bar{t}}}{1-R}=\nu
\end{aligned}
$$

${ }^{42}$ In the $\log$ case, $\varepsilon=1, R=r<1, f_{<}=f_{>}$and the solution derived below extends to this case. 
Using $\frac{K_{0}^{\mu-1} f_{<}^{\phi}}{1-\delta}=\frac{\nu}{1-\alpha}$ from the expression of $K_{0}$, we have from last equation:

$$
f_{>}^{\phi} K_{\bar{t}}^{\mu-1} \delta^{-\mu \bar{t}} \frac{R^{\bar{t}}}{1-R}=\frac{K_{0}^{\mu-1} f_{<}^{\phi}}{1-\delta}
$$

Injecting (G.6) and (G.8) into this:

$$
\left(\left(F-f_{<} \frac{1-r^{\bar{t}}}{1-r}\right) \frac{1-R}{R^{\bar{t}}}\right)^{\frac{\phi}{\mu-1}}\left(\frac{\bar{M}}{K_{0}}-a\right)=\left(\frac{f_{<}^{\phi}}{1-\delta} \frac{1-R}{R^{\bar{t}}} \delta^{\mu \bar{t}}\right)^{\frac{1}{\mu-1}}
$$

Combining this with (G.9) we have an equation in $f_{<}$:

$$
\begin{aligned}
f_{<}^{\frac{\phi-1}{\mu}-\frac{\phi}{\mu-1}}\left(\left(F-f_{<} \frac{1-r^{\bar{t}}}{1-r}\right) \frac{1-R}{R^{\bar{t}}}\right)^{\frac{\phi}{\mu-1}-\frac{\phi-1}{\mu}} & =\delta^{-\bar{t}}\left(\frac{\delta^{\mu \bar{t}}}{1-\delta} \frac{1-R}{R^{\bar{t}}}\right)^{\frac{1}{\mu-1}} \\
\left(\frac{F}{f_{<}}-\frac{1-r^{\bar{t}}}{1-r}\right) \frac{1-R}{R^{\bar{t}}} & =b^{\mu}
\end{aligned}
$$

where $b:=\left(\frac{\delta^{\bar{t}}}{1-\delta} \frac{1-R}{R^{t}}\right)^{\frac{1}{\mu+\phi-1}}$.

$$
f_{<}=\left(b^{\mu} \frac{R^{\bar{t}}}{1-R}+\frac{1-r^{\bar{t}}}{1-r}\right)^{-1} F=\frac{1-\delta}{a+c} F
$$

where:

$$
c:=b^{\mu} \frac{R^{\bar{t}}}{1-R}(1-\delta)=\left(\frac{1-\delta}{\delta^{\bar{t}}} \frac{R^{\bar{t}}}{1-R}\right)^{\frac{-\mu}{\mu+\phi-1}} \frac{R^{\bar{t}}}{1-R}(1-\delta)=\left((1-\delta) \frac{R^{\bar{t}}}{1-R}\right)^{\frac{\phi-1}{\mu+\phi-1}} \delta^{\frac{\mu \bar{t}}{\mu+\phi-1}}
$$

Injecting (G.12) into (G.9) (at the second line), we deduce $K_{0}$ :

$$
\begin{aligned}
K_{0} & =\bar{M}\left(f_{<}^{\frac{\phi-1}{\mu}}\left(\left(F-f_{<} \frac{1-r^{\bar{t}}}{1-r}\right) \frac{1-R}{R^{\bar{t}}}\right)^{-\frac{\phi-1}{\mu}} \delta^{\bar{t}}+a\right)^{-1} \\
& =\frac{\bar{M}}{b^{1-\phi} \delta^{\bar{t}}+a}=\frac{\bar{M}}{a+c}
\end{aligned}
$$

Injecting (G.14) into (G.8), we deduce $K_{\bar{t}}$ :

$$
K_{\bar{t}}=\frac{c \bar{M}}{a+c}=c K_{0}=b^{1-\phi} \delta^{\bar{t}} K_{0}
$$

We get $f_{>}$from (G.6) and (G.13):

$$
f_{>}=\frac{1-R}{R^{\bar{t}}}\left(F-f_{<} \frac{1-r^{\bar{t}}}{1-r}\right)=b^{\mu} f_{<}
$$


Finally, the inter-temporal welfare writes:

$$
\begin{aligned}
W_{\bar{t}} & =\frac{f_{0}^{\phi} m_{0}^{\mu}}{1-\varepsilon}+\sum_{x=1}^{\bar{t}-1}(1+\rho)^{-x} \frac{f_{x}^{\phi} K_{x}^{\mu}}{1-\varepsilon}+(1+\rho)^{-\bar{t}} \frac{f_{\bar{t}}^{\phi} K_{\bar{t}}^{\mu}}{1-\varepsilon}+\sum_{x>\bar{t}}(1+\rho)^{-x} \frac{f_{x}^{\phi} K_{\bar{t}}^{\mu}}{1-\varepsilon} \delta^{\mu(x-\bar{t})} \\
& =\frac{1}{1-\varepsilon}\left(f_{<}^{\phi} K_{0}^{\mu}+\sum_{x=1}^{\bar{t}-1}(1+\rho)^{-x} f_{<}^{\phi} r^{\phi x} K_{0}^{\mu} r^{\mu x}+(1+\rho)^{-\bar{t}} f_{>}^{\phi} R^{\phi \bar{t}} K_{\bar{t}}^{\mu}+\sum_{x>\bar{t}}(1+\rho)^{-x} f_{>}^{\phi} R^{\phi x} K_{\bar{t}}^{\mu} \delta^{\mu(x-\bar{t})}\right) \\
& =\frac{1}{1-\varepsilon}\left(f_{<}^{\phi} K_{0}^{\mu} \frac{1-\left(\frac{r^{\phi+\mu}}{1+\rho}\right)^{\bar{t}}}{1-\frac{r^{\phi+\mu}}{1+\rho}}+(1+\rho)^{-\bar{t}} b^{\mu \phi} f_{<}^{\phi} R^{\phi \bar{t}} b^{\mu(1-\phi)} \delta^{\mu \bar{t}} K_{0}^{\mu}+f_{>}^{\phi} K_{\bar{t}}^{\mu} \delta^{-\mu \bar{t}} \frac{R^{\bar{t}+1}}{1-R}\right) \\
& =\frac{1}{1-\varepsilon}\left(f_{<}^{\phi} K_{0}^{\mu}\left(\frac{1-\left(\frac{r^{\phi+\mu}}{1+\rho}\right)^{\bar{t}}}{1-\frac{r^{\phi+\mu}}{1+\rho}}+b^{\mu} \delta^{\mu \bar{t}}\left(R^{\phi \bar{t}}(1+\rho)^{-\bar{t}}+\delta^{-\mu \bar{t}} \frac{R^{\bar{t}+1}}{1-R}\right)\right)\right) \\
& =\frac{1}{1-\varepsilon}\left(F^{\phi} \bar{M}^{\mu}\left(\frac{1-\delta}{a+c}\right)^{\phi}(a+c)^{-\mu}\left(\frac{1-r^{\bar{t}}}{1-r}+b^{\mu} \frac{R^{\bar{t}}}{1-R}\right)\right) \\
& =\frac{1}{1-\varepsilon}\left(F^{\phi} \bar{M}^{\mu}(1-\delta)^{\phi-1}(a+c)^{1-\phi-\mu}\right)
\end{aligned}
$$

\section{H On-line Appendix: Welfare analysis}

In case (2.a) of Proposition 1 the inter-temporal welfare takes the value:

$$
\begin{aligned}
W_{\infty} & =\sum_{t \geq 0}(1+\rho)^{-t} \frac{f_{t}^{\phi} K_{t}^{\mu}}{1-\varepsilon} \\
& =\frac{f_{0}^{\phi} m_{0}^{\mu}}{1-\varepsilon}+\sum_{t \geq 1}(1+\rho)^{-t} \frac{f_{t}^{\phi} K_{t}^{\mu}}{1-\varepsilon} \\
& =\frac{f_{0}^{\phi} m_{0}^{\mu}}{1-\varepsilon}+\frac{f_{0}^{\phi} m_{0}^{\mu}}{1-\varepsilon} \sum_{t \geq 1}\left(\frac{r^{\phi+\mu}}{1+\rho}\right)^{t} \\
& =\frac{f_{0}^{\phi} m_{0}^{\mu}}{1-\varepsilon}\left(1+\frac{r}{1-r}\right) \\
& =\frac{F^{\phi} M^{\mu}}{1-\varepsilon}(1-r)^{\phi+\mu-1}(1-\delta)^{-\mu}
\end{aligned}
$$

In the previous computations, we used $\frac{r^{\phi+\mu}}{1+\rho}=(1+\rho)^{\frac{\phi+\mu}{\phi+\mu-1}-1}=(1+\rho)^{\frac{1}{\phi+\mu-1}}=r$. It follows that:

$$
\frac{\partial W_{\infty}}{\partial \delta}=F^{\phi} M^{\mu}(1-r)^{\phi+\mu-1}(1-\alpha)(1-\delta)^{-\mu-1}>0
$$

In case (1) of Proposition 1 the inter-temporal welfare takes the value:

$$
\begin{aligned}
W_{0} & =\sum_{t \geq 0} \frac{(1+\rho)^{-t}}{1-\varepsilon} f_{t}^{\phi} m_{0}^{\mu} \delta^{\mu t} \\
& =\frac{1}{1-\varepsilon} M^{\mu} F^{\phi}(1-R)^{\phi} \sum_{t \geq 0}(1+\rho)^{-t} \delta^{\mu t} R^{\phi t} \\
& =\frac{1}{1-\varepsilon} M^{\mu} F^{\phi}(1-R)^{\phi-1}
\end{aligned}
$$


In the computations, we used $(1+\rho)^{-1} \delta^{\mu} R^{\phi}=R^{1-\phi} R^{\phi}=R$. It follows that

$$
\begin{aligned}
\frac{\partial W_{0}}{\partial \delta} & =\frac{1-\phi}{1-\varepsilon} M^{\mu} F^{\phi}(1-R)^{\phi-2} \frac{\partial R}{\partial \delta} \\
& =\left\{\begin{array}{l}
(+) \cdot(+)>0 \text { for } \varepsilon \in(0,1) \\
(-) \cdot(-)>0 \text { for } \varepsilon>1
\end{array}\right.
\end{aligned}
$$

Welfare increases with $\delta$ also in case (2.b) since it is a mix of the two cases above. 